\title{
Chemistry-climate model SOCOL: a validation of the present-day climatology
}

\author{
T. Egorova ${ }^{1,2}$, E. Rozanov ${ }^{1,2}$, V. Zubov ${ }^{3}$, E. Manzini ${ }^{4}$, W. Schmutz ${ }^{2}$, and T. Peter ${ }^{1}$ \\ ${ }^{1}$ Institute for Atmospheric and Climate Science ETH, Zurich, Switzerland \\ ${ }^{2}$ Physical-Meteorological Observatory/World Radiation Center, Davos, Switzerland \\ ${ }^{3}$ Main Geophysical Observatory, St.-Petersburg, Russia \\ ${ }^{4}$ National Institute for Geophysics and Volcanology, Bologna, Italy \\ Received: 15 December 2004 - Published in Atmos. Chem. Phys. Discuss.: 1 February 2005 \\ Revised: 2 May 2005 - Accepted: 9 May 2005 - Published: 21 June 2005
}

\begin{abstract}
In this paper we document "SOCOL", a new chemistry-climate model, which has been ported for regular PCs and shows good wall-clock performance. An extensive validation of the model results against present-day climate data obtained from observations and assimilation data sets shows that the model describes the climatological state of the atmosphere for the late 1990s with reasonable accuracy. The model has a significant temperature bias only in the upper stratosphere and near the tropopause at high latitudes. The latter is the result of the rather low vertical resolution of the model near the tropopause. The former can be attributed to a crude representation of radiation heating in the middle atmosphere. A comparison of the simulated and observed link between the tropical stratospheric structure and the strength of the polar vortex shows that in general, both observations and simulations reveal a higher temperature and ozone mixing ratio in the lower tropical stratosphere for the case with stronger Polar night jet (PNJ) and slower Brewer-Dobson circulation as predicted by theoretical studies.
\end{abstract}

\section{Introduction}

Forecasting future ozone and climate changes is among the most pressing and challenging problems in contemporary environmental science. The Earth's climate is determined by a variety of physical and chemical processes within a complex system reacting to various external forcings, as well as by short-term and long-term internal variability (IPCC, 2001). Therefore, projections of the atmospheric state can be made only by means of sophisticated modeling tools, which are able to represent all relevant atmospheric physical, chemical and dynamical processes and their interactions. During

Correspondence to: T. Egorova

(t.egorova@pmodwrc.ch) the previous decade the development of such tools was substantially advanced reflecting the need for reliable climate and ozone layer forecasting on the one hand and the tremendous growth of computational capabilities on the other hand. These advances lead to the development of General Circulation Models (GCMs) to which interactive chemistry has been added, the so-called Chemistry-Climate Models (CCMs) (see Austin et al. (2003), and references therein). Each of these models is able to simulate all relevant physical, dynamical and chemical processes in 3-dimensional space and their evolution in time. However, when applied to the simulation of future climate changes and ozone recovery in the $21^{\text {th }}$ century these models may produce rather different results (e.g., Austin et al. (2003)). For example, the GISS CCM (Shindell et al., 1998) predicted a delay of ozone recovery over the Arctic due to the influence of greenhouse gases (GHG), while the DLR CCM (Schnadt et al., 2002) predicted acceleration and the CCSR/NIES CCM (Nagashima et al., 2002) did not show any changes in ozone recovery under changing climate conditions. Resolving this controversy requires more attention to extensive model validation.

Due to non-linearity in the atmospheric processes CCMs produce "realistic" rather than "real" atmospheric states, therefore the model cannot be validated simply by day-to-day comparisons between CCM output and observations. Validation can only be made in terms of the models ability to reproduce (1) the climatological mean state of the atmosphere (2) any trends with respect to that climatology (3) an accurate representation of observed atmospheric variability and processes. Each of these three steps in model validation is not straightforward and has their own caveats, mostly because our knowledge of atmospheric climatology and processes is incomplete. In this paper we concentrate on the model validation with regard to the first step.

At the moment we have a great deal of information about the global present-day atmosphere from the last 25 years

(C) 2005 Author(s). This work is licensed under a Creative Commons License. 
of intensive satellite measurements, but only limited knowledge about potential variability in global atmospheric parameters before this period. On the other hand, there is evidence from historical studies (e.g., Brönnimann et al., 2004) that atmospheric variability could have been much larger in the past than in the present day atmosphere. Therefore, the present day climatology obtained mainly from satellite observations should be considered as only one particular realization of a general sequence. Deviations of a simulated climatology from the particular observed climatology, or even from a particular reality (namely the one assumed by planet Earth), should be interpreted with caution. These deviations between model and reality must be evaluated to determine where they are significant, i.e. where the discrepancies cannot be explained in terms of a system anomaly. The particular locations, time periods, physical quantities or relationships, where significant deviations occur, might be called "hotspots". In practical terms a determination of "hotspots" may be rather difficult simply because we often do not know the statistical properties of the real atmosphere for certain conditions. For the validation of a model climatology one usually applies assimilated data products (e.g. Butchart and Austin, 1998; Pawson et al., 2000). These data sets are the results of simulations with a comprehensive model running in assimilation mode, e.g. a numerical weather prediction model with a variety of available observations integrated into the model to enable better representation of the mean state of the atmosphere and its variability. The various assimilation schemes and applied models differ substantially and provide alternative atmospheric states, which can be considered as different realizations of the contemporary climate. This variability together with interannual variability of the observed and simulated meteorological fields provides a basis to estimate the significance of the model errors and define model "hotspots", i.e. regions in space and time where the model deficiency is the most pronounced and significant. The model validation can be also performed using direct satellite (e.g. Rozanov et al., 2001, Steil et al, 2003) or ground based (Struthers et al. 2004) measurements of the chemical species. In this case the significance of the model deviation from the observations can be estimated using standard deviations of the simulated and observed fields.

Recently, process-oriented validation of CCMs has gained a lot of attention because this approach opens new opportunities to validate models. This kind of validation was designed to reinforce the standard comparisons considering model abilities to reproduce atmospheric processes in comparison with observations (Austin et al. 2003, Eyring et al., 2004). Here we present an example of process-oriented validation that we believe can be used to validate CCMs, namely the comparison of the simulated and observed relationship of stratospheric ozone and temperature with the strength of the northern polar vortex during boreal winter. The aim of this particular exercise is to validate the ability of SOCOL to simulate the relationship between the strength of the polar vortex and stratospheric ozone and temperature during the boreal winter. It is well known that the positive phase of the $\mathrm{AO}$ is characterized by a deeper vortex and more intensive Polar Night Jet (e.g., Thompson and Wallace, 1998). Therefore, it is theoretically expected (e.g., Kodera and Kuroda, 2002) that the positive $\mathrm{AO}$ phase results in a weaker meridional circulation and consequently leads to warmer temperatures and elevated ozone in the tropical lower stratosphere. Here we attempt to find these features in the observational data and model simulations and compare them.

In this paper we present the description and validation of the present day climatology of the new chemistryclimate model SOCOL (modeling tool for studies of SOlar Climate Ozone Links) that has been developed at Physical and Meteorological Observatory/World Radiation Center (PMOD/WRC), Davos in collaboration with ETH Zürich and MPI Hamburg. The meteorological and chemical fields generated by the model are compared with the data obtained from different assimilation products and model "hotspots" are defined.

The layout of this paper is as follows: In Sect. 2 we describe SOCOL and the design of the runs performed with it, in Sect. 3 we describe data that we used for model validation, and in Sect. 4 we present the results of the model validation. In particular, we show the deviation of the simulated meteorological fields from the observations and their significance. We also present a comparison of the simulated total ozone and other species with satellite measurements and illustrate the sensitivity of the ozone and temperature to the strength of the northern polar vortex during boreal winter. The last section presents our conclusions.

\section{Description of the Chemistry-Climate Model SOCOL}

The chemistry-climate model SOCOL has been developed as a combination of a modified version of the MA-ECHAM4 GCM (Middle Atmosphere version of the "European Center/Hamburg Model 4" General Circulation Model) and a modified version of the UIUC (University of Illinois at Urbana-Champaign) atmospheric chemistry-transport model MEZON described in detail by Rozanov et al. $(1999,2001)$ and Egorova et al. $(2001,2003)$.

\subsection{GCM component}

MAECHAM4 is the middle atmosphere GCM developed at the MPI for Meteorology in Hamburg (Manzini et al., 1997; Charron and Manzini, 2002) based on the standard ECHAM4 GCM (Roeckner et al, 1996). The ECHAM GCMs evolve originally from the spectral weather prediction model of ECMWF (Simmons et al., 1989). It is a spectral model with T30 horizontal truncation resulting in a grid spacing of about 
$3.75^{\circ}$; in the vertical direction the model has 39 levels in a hybrid sigma-pressure coordinate system spanning the model atmosphere from the surface to $0.01 \mathrm{hPa}$; a semi-implicit time stepping scheme with weak filter is used with a time step of $15 \mathrm{~min}$ for dynamical processes and physical process parameterizations; full radiative transfer calculations are performed every 2 hours, but heating and cooling rates are calculated every $15 \mathrm{~min}$. The radiation scheme is based on the ECMWF radiation code (Fouquart and Bonnel, 1980; Morcrette, 1991). The orographic gravity wave parameterization is based on the formulation of McFarlane (1987). The parameterization of momentum flux deposition due to a continuous spectrum of vertically propagating gravity waves follows Hines (1997a, b), and the implementation of the Doppler spread parameterization is according to Manzini et al. (1997). A more detailed description of MA-ECHAM4 can be found in Manzini and McFarlane (1998), and references therein. With respect to the standard MA-ECHAM4, the gravity wave source spectrum of the Doppler spread parameterization has been modified. Namely, the current model version uses a spatially and temporally constant gravity wave parameter for the specification of the source spectrum, as in case UNI2 of Charron and Manzini (2002). Therefore, an isotropic spectrum with a gravity wave wind speed of $1 \mathrm{~m} / \mathrm{s}$ and an effective wave number $\mathrm{K}^{*}=2 \pi(126 \mathrm{~km})^{-1}$ is launched from the lower troposphere, at about $600 \mathrm{hPa}$.

\subsection{CTM component}

The chemical-transport part MEZON (Model for the Evaluation of oZONe trends) simulates 41 chemical species $\left(\mathrm{O}_{3}\right.$, $\mathrm{O}\left({ }^{1} \mathrm{D}\right), \mathrm{O}\left({ }^{3} \mathrm{P}\right), \mathrm{N}, \mathrm{NO}, \mathrm{NO}_{2}, \mathrm{NO}_{3}, \mathrm{~N}_{2} \mathrm{O}_{5}, \mathrm{HNO}_{3}, \mathrm{HNO}_{4}$, $\mathrm{N}_{2} \mathrm{O}, \mathrm{H}, \mathrm{OH}, \mathrm{HO}_{2}, \mathrm{H}_{2} \mathrm{O}_{2}, \mathrm{H}_{2} \mathrm{O}, \mathrm{H}_{2}, \mathrm{Cl}, \mathrm{ClO}, \mathrm{HCl}, \mathrm{HOCl}$, $\mathrm{ClNO}_{3}, \mathrm{Cl}_{2}, \mathrm{Cl}_{2} \mathrm{O}_{2}, \mathrm{CF}_{2} \mathrm{Cl}_{2}, \mathrm{CFCl}_{3}, \mathrm{Br}, \mathrm{BrO}, \mathrm{BrNO}_{3}, \mathrm{HOBr}$, $\mathrm{HBr}, \mathrm{BrCl}, \mathrm{CBrF}_{3}, \mathrm{CO}, \mathrm{CH}_{4}, \mathrm{CH}_{3}, \mathrm{CH}_{3} \mathrm{O}_{2}, \mathrm{CH}_{3} \mathrm{OOH}$, $\mathrm{CH}_{3} \mathrm{O}, \mathrm{CH}_{2} \mathrm{O}$, and $\mathrm{CHO}$ ) from the oxygen, hydrogen, nitrogen, carbon, chlorine and bromine groups, which are determined by 118 gas-phase reactions, 33 photolysis reactions and 16 heterogeneous reactions on/in sulfate aerosol (binary and ternary solutions) and polar stratospheric cloud (PSC) particles (Carslaw et al., 1995). The mixing ratio of source gases for chlorines and bromines have been scaled in the near surface air to take into account the other important sources. The diagnostic thermodynamic scheme for the calculation of the condensed phase content of PSCs also makes use of the vapor pressure of nitric acid trihydrate (NAT) following Hanson and Mauersberger (1988). The PSC scheme uses pre-described cloud particle number densities and assumes the cloud particles to be in thermodynamic equilibrium with their gaseous environment. It allows the description of the condensation and evaporation of the PSC without detailed microphysical calculations. Sedimentation of NAT and ice (type I and II) PSC particles is described according to the approach proposed by Butchart and Austin (1996). The chemical solver is based on the implicit iterative
Newton-Raphson scheme (Ozolin, 1992; Stott and Harwood, 1993). The basic routine of the solver has been accelerated to improve its computational performance. A special acceleration technique for solving a sparse system of linear algebraic equations was developed and used. This technique utilizes the following main ideas: (1) the algorithm of the LU-decomposition/back-substitution of the Jacobian matrix is modified to include only nonzero operations (LU denotes the lower/upper triangular matrix decomposition regularly used in numerical analysis to solve a system of linear equations); (2) the Jacobian matrix is rearranged according to the number of nonzero elements in the row: this rearranging allows minimization of the number of the nonzero calculations during the LU decomposition/back-substitution process; and (3) the sequence of rows of the Jacobian matrix depends only on the photochemical reaction table used in the model and is the same for all grid cells of the model domain (Sherman and Hindmarsh, 1980; Jacobson and Turco, 1994). The reaction coefficients are taken from DeMore et al. (1997) and Sander et al. (2000). The photolysis rates are calculated at every step using a look-up-table approach (Rozanov et al., 1999). The transport of all considered species is calculated using the hybrid numerical advection scheme of Zubov et al. (1999). The transport scheme is a combination of the Prather scheme (Prather, 1986), which is used in the vertical direction, and a semi-Lagrangian (SL) scheme, which is used for horizontal advection on a sphere (Williamson and Rasch, 1989). The use of the Prather scheme ensures good representation of concentration gradients in the vertical direction. The SL scheme for the horizontal transport allows a significantly larger time step even near the poles where the sizes of the grid cells are smaller. Furthermore, use of the Prather scheme for transport in only one dimension (instead of three) reduces the number of moments that define the distribution of species in each model grid box from 10 to 3 . Thus, the combination of the SL scheme with the Prather scheme yields a significant gain in economy in the transport calculations compared with using the Prather scheme alone, while attaining accuracy higher than that of the SL scheme alone. A detailed description of the design and performance of the hybrid transport scheme based on simple analytical tests is given by Zubov et al. (1999). The species are transported in the troposphere by the model winds and by vertical eddy diffusion (Rozanov et al., 1999). The deposition velocities of $\mathrm{CO}, \mathrm{NO}_{\mathrm{x}}, \mathrm{HNO}_{3}, \mathrm{O}_{3}$ and $\mathrm{H}_{2} \mathrm{O}_{2}$ are prescribed for different types of surface following Müller and Brasseur (1995). MEZON has been extensively validated against observations in off-line mode, driven by UKMO meteorological fields (Rozanov et al., 1999; Egorova et al., 2003) and in on-line mode as a part of UIUC CCM (Rozanov et al., 2001). It has been coupled to different GCMs to study Pinatubo aerosol effects (Rozanov et al., 2002a) and influence of 11-year solar variability influence on global climate and photochemistry (Rozanov et al., 2004; Egorova et al., 2004). 
Table 1. Climatological data sets used for model validation.

\begin{tabular}{lll}
\hline Data source & Time period used & Upper level \\
\hline UKMO & $1992-1999(8$ years $)$ & $0.3 \mathrm{hPa}$ \\
CPC & $1991-1998$ ( 8 years $)$ & $1 \mathrm{hPa}$ \\
NCEP & $1991-1999$ (9 years $)$ & $10 \mathrm{hPa}$ \\
ERA-40 & $1991-2000(10$ years $)$ & $1 \mathrm{hPa}$ \\
TOVS & $1991-1996$ (6 years $)$ & $1 \mathrm{hPa}$ \\
URAP & $1992-1999(8$ years $)$ & $0.01 \mathrm{hPa}$ \\
\hline
\end{tabular}

\subsection{GCM-CTM interface}

For the coupling with MA-ECHAM4, MEZON has been improved to take into account the latest revisions of the chemical reaction constants. Several photolytic and gas-phase reactions that are potentially important for mesospheric chemistry have been added to the model. The new scheme for photolysis rate calculations spans the spectral region 120 $750 \mathrm{~nm}$ divided into 73 spectral intervals and now specifically includes the Lyman- $\alpha$ line and the Schumann-Runge continuum. We have tested its performance using a 1-D chemistry-climate model (Rozanov et al., 2002b). The GCM and CTM components of SOCOL are coupled via the threedimensional fields of wind, temperature, ozone and water vapor. The GCM provides the horizontal and vertical winds, temperature and tropospheric humidity for the CTM, which returns 3-D fields of the ozone and water vapor mixing ratios back to the GCM in order to calculate radiation fluxes and heating rates. The water cycle in the troposphere and lower stratosphere is treated in the GCM part of the model, while the water vapor chemistry and transport in the stratosphere and mesosphere is treated in chemistry-transport part of the model. The unique water vapor field is transferred from GCM to CTM and back at every step.

\subsection{Community model}

To make SOCOL available for a wide scientific community we ported the entire CCM on desktop personal computers (PCs). A 10-year long simulation takes about 40 days of wall-clock time on a PC with a processor running at $2.5 \mathrm{GHz}$, which allows the performance of multiyear integrations. The simultaneous use of several PCs allows the performance of ensemble calculations with ease. Reasonable model performance and availability of personal desktop computers makes SOCOL available for application by scientific groups around the world without access to large super-computer facilities, opening wide perspectives for model exploitation and improvement. The technical information is given at the end of the paper.

\subsection{Model set-up}

As a first step toward the validation of SOCOL we have carried out a 40-year long control run for present day conditions. For this run we used sea surface temperature and sea ice (SST/SI) distributions prescribed from AMIP II monthly mean distributions, which are averages from 1979 to 1996 (Gleckler, 1996). The lower boundary conditions for the source gases have been prescribed following Rozanov et al. (1999) and are representative for conditions of 1995. We use prescribed mixing ratios in the planetary boundary layer for the source gases and prescribed fluxes of $\mathrm{CO}$ and $\mathrm{NO}_{\mathrm{x}}$ from the surface, airplanes and lightning, similar to Rozanov et al. (1999). The mixing ratio of $\mathrm{CO}_{2}$ is set to $356 \mathrm{ppmv}$ everywhere. The initial distributions of the meteorological quantities and gas mixing ratios have been adopted from MA-ECHAM4 and from an 8-year long Stratospheric CTM run (Rozanov et al., 1999). Later on in this paper we will analyze the 40 -year mean of the simulated quantities.

\section{Description of the data used for validation}

To validate the large-scale atmospheric behavior of the SOCOL model and to specify the significance of the model errors we use data sets for the middle atmosphere which are the results of the efforts of different meteorological institutions around the world: the European Center for Medium Range Weather Forecast (ECMWF), United Kingdom Meteorological Office (UKMO), National Center of Environmental Predictions (NCEP) and Climate Prediction Center (CPC) reanalysis projects. We have also used monthly mean data obtained from the TOVS instrument. All data sets have been downloaded from the SPARC Data Center (http://www. sparc.sunysb.edu). Detailed descriptions of the data sets used have been presented in the SPARC inter-comparison project of the middle atmosphere (SPARC, 2002). Some characteristic parameters of the applied data sets are summarized in Table 1. According to the SPARC comparison report (SPARC, 2002) UKMO, CPC and NCEP are warm biased in the tropical tropopause area by $2-3 \mathrm{~K}$, while UKMO has a warm bias in the upper stratosphere up to $5 \mathrm{~K}$. To estimate the significance of the deviation of the simulated climatology from the observed climatology we have combined all data sets listed in Table 1 (except URAP data) in one data set. In doing this we obtained 41 consecutive years of observational data for the validation (only 32 include data above $10 \mathrm{hPa}$ ). From this extended data set we have calculated a monthly mean climatology of the zonal wind and temperature, as well as the standard deviation of these quantities, which includes the interannual variability as well as variability due to differences between data sets. To validate the model winds in the mesosphere we used URAP (http://code916.gsfc.nasa.gov) zonal mean zonal wind, which is a combination of UKMO winds in the stratosphere and the High Resolution Doppler 

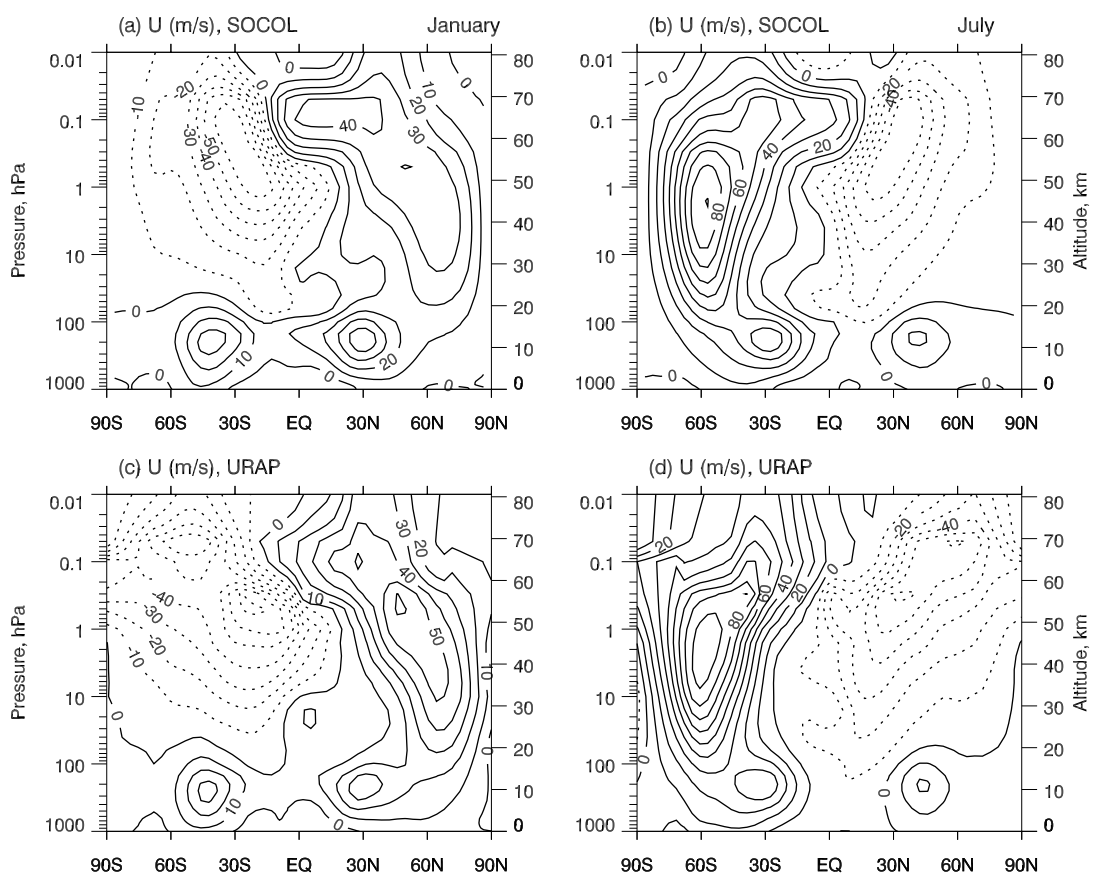

Fig. 1. Meridional cross-section of the zonal mean zonal wind $\left(\mathrm{ms}^{-1}\right)$ for January (left panel) and July (right panel): (a, b) simulated, (c, d) observed. Observed values are from URAP database.

Imager (HRDI) winds in the mesosphere (Swinbank and Ortland, 2003).

Total ozone data have been taken from merged TOMS/SBUV data set (http://code916.gsfc.nasa.gov/Data services/merged/mod_data.public.html) and averaged over 10 years (1991-2000). For the comparison of ozone $\left(\mathrm{O}_{3}\right)$, water vapor $\left(\mathrm{H}_{2} \mathrm{O}\right)$, methane $\left(\mathrm{CH}_{4}\right)$ and $\mathrm{HCl}$ in the stratosphere we used the URAP data set (http://code916.gsfc.nasa. gov) that provides a comprehensive description of the reference stratosphere from the data recorded by several instruments onboard of UARS. Because we used only one data set for every considered species the standard deviation in this case is defined only by interannual variability. For all considered quantities the differences of the simulated and observed fields are considered as significant if they exceed the sum of the standard deviations of the simulated and observed fields. To analyze the response of the atmosphere to the strength of the polar vortex we use the same 40-year long simulation of the present day atmosphere. We divided the simulated data into two groups according to the phase of Arctic Oscillation during the boreal winter season defined from the principal component of the first EOF of the geopotential height field at $70 \mathrm{hPa}$ and contrasted the difference between these two groups against observational data processed in an identical way. The statistical significance of this difference is estimated using the well known Student's t-test. The observations we used are NMC data (for 1978-1998) and SAGE I/II ozone density (for 1979-2001) compiled by W. Randel et al. (www.acd.ucar.edu/ $\sim$ randel).

\section{Results}

4.1 Monthly mean zonal mean zonal wind and temperature

\subsubsection{Temperature and wind fields}

Monthly means of zonally averaged zonal winds for January and July are presented in Fig. 1 in comparison with the 8year means of the same quantities acquired from the URAP data sets. The model reproduces all the main climatological features of the observed zonal wind distribution qualitatively, and with a few exceptions even quantitatively. The separation of the stratospheric and tropospheric westerly jets is well simulated by SOCOL. The tropospheric subtropical jets, their shape and location are in good agreement as is the polar night jet (PNJ) core, in the middle and upper stratosphere. However, for January in the Northern Hemisphere the intensity of the tropospheric subtropical jet is overestimated by about $10 \mathrm{~ms}^{-1}$. The PNJ's intensity is underestimated by the same amount, and its maximum is located at higher altitudes than in the URAP data. SOCOL captures the observed equatorward tilt of the stratospheric westerly core. The most noticeable disagreement occurs in the lower mesosphere, where the simulated easterly winds do not penetrate to the high-latitude area over the summer hemisphere. It should be noted that the model does not reproduce the QBO in the equatorial atmosphere, which can affect the simulated climatology of the temperature, winds and chemical species (Giorgetta et al., 2004). 

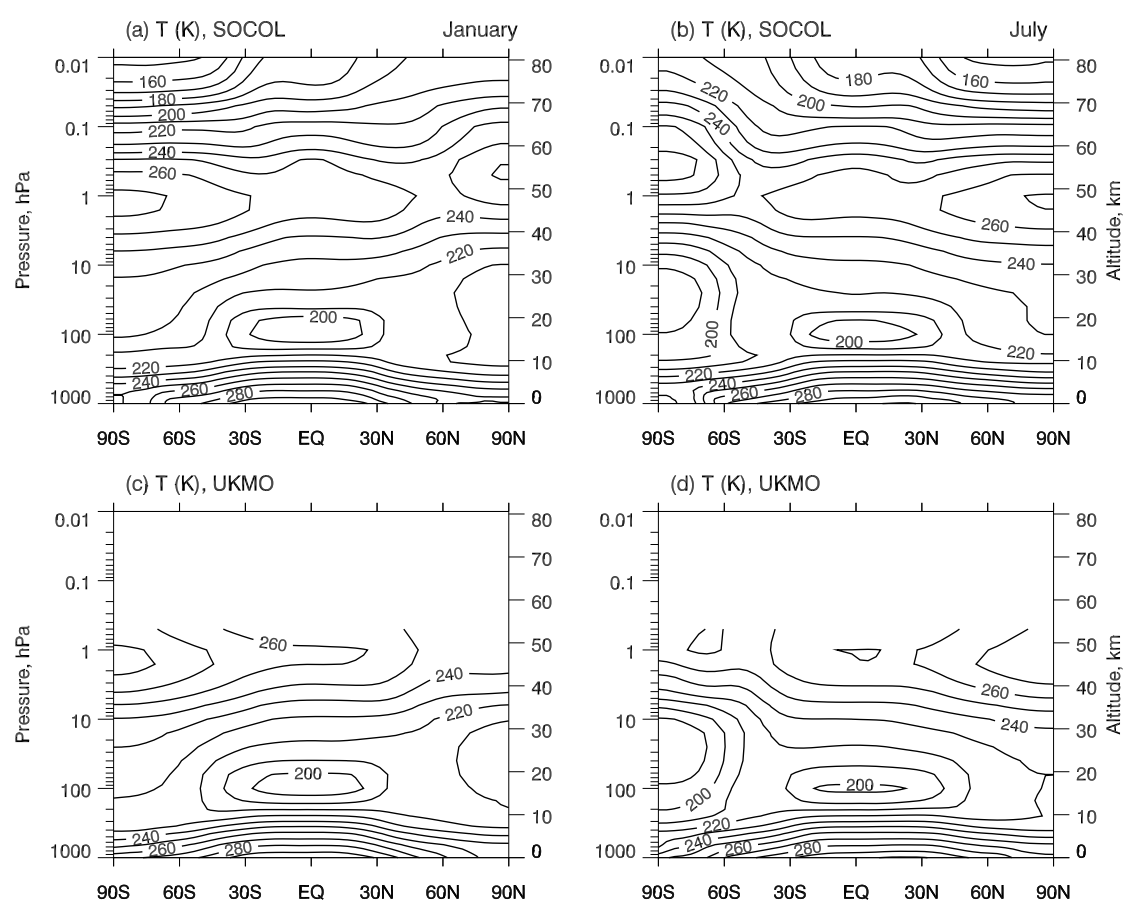

Fig. 2. Same as Fig. 1 but for the zonal mean temperature (K). Observed values are from UKMO reanalysis.
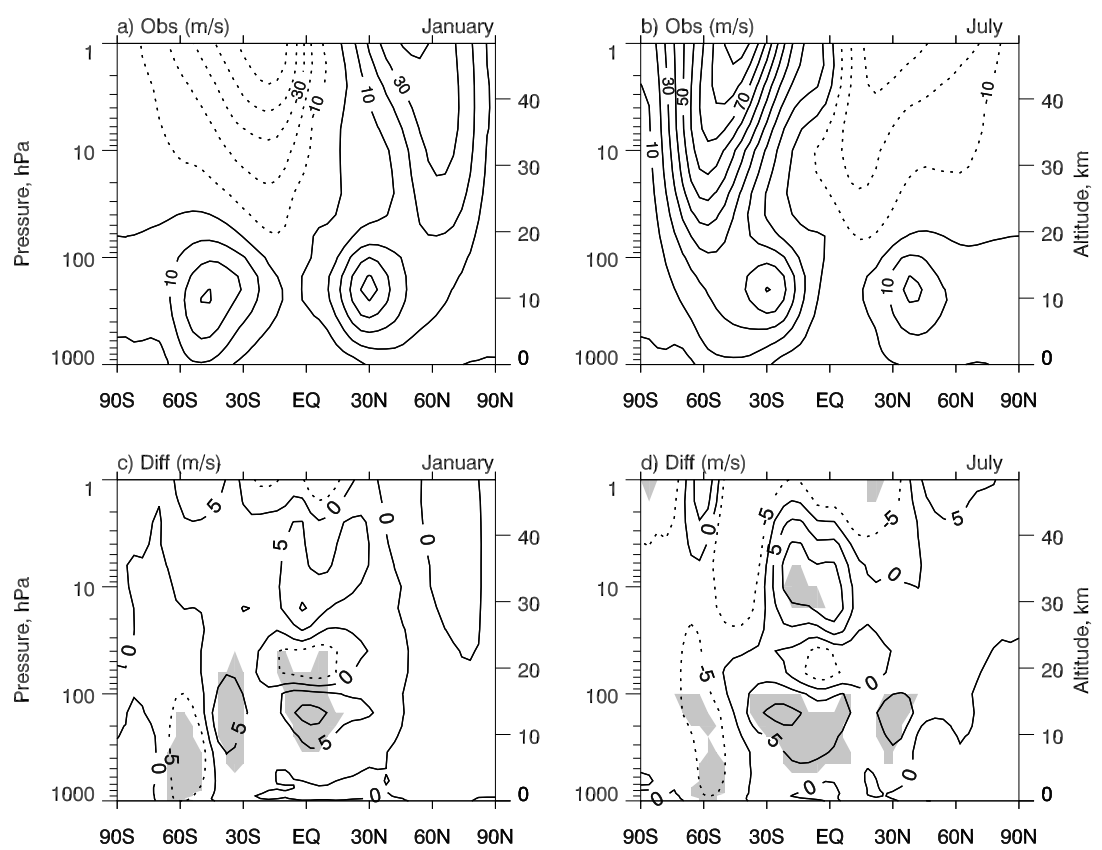

Fig. 3. Zonal mean zonal wind for January and July from the observation data composite (a, b) (contours in steps of $10 \mathrm{~ms}^{-1}$ ) and difference (contours in steps of $5 \mathrm{~ms}^{-1}$ ) between simulated and observed data (c, d). Shading marks "hotspots", the area where the deviations are significant.

Figure 2 presents a comparison of latitude-pressure crosssections of simulated and UKMO zonal mean temperatures for January and July. The evaluation of the temperature distribution reveals that in the lower stratosphere the general agreement of the location and magnitude of the simulated extremes is rather good. SOCOL reproduces the main observed features of zonal mean temperature distribution well: warm troposphere, cold tropical tropopause without apparent bias, 

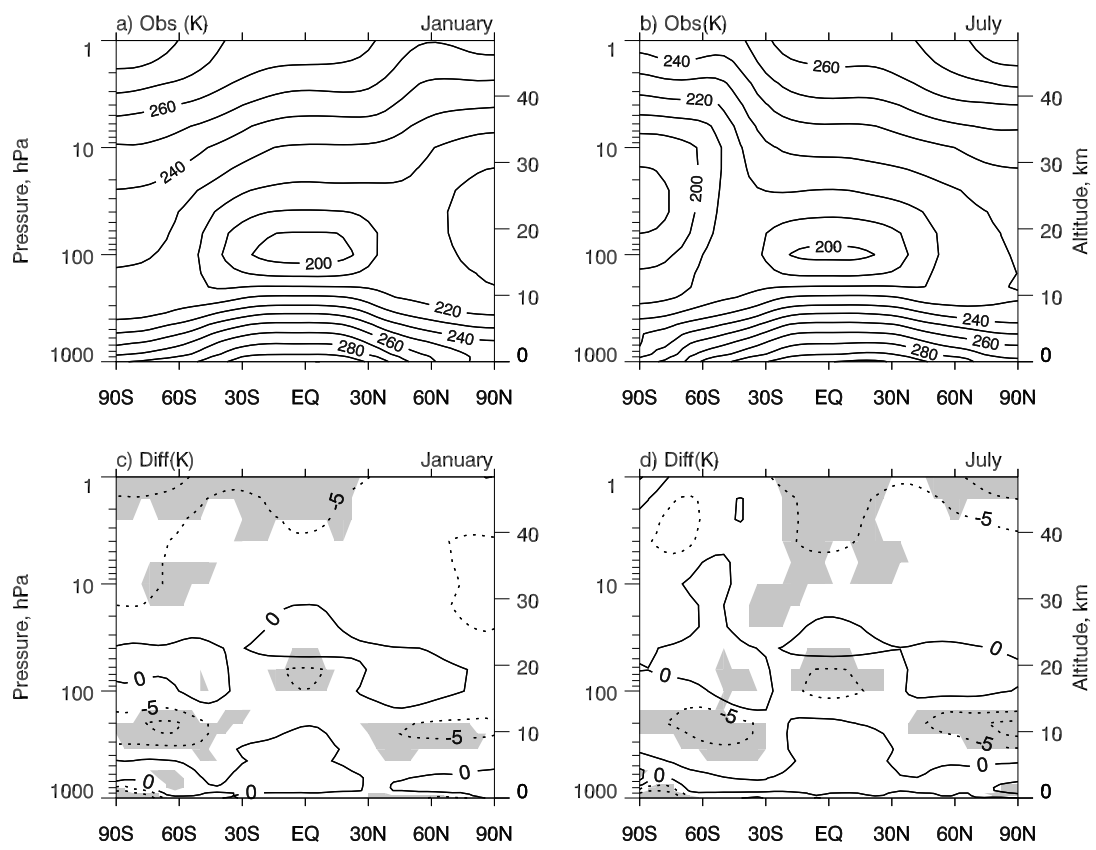

Fig. 4. Same as for Fig. 3, but for temperature (contours in steps of $10 \mathrm{~K}$ and $5 \mathrm{~K}$ accordingly).
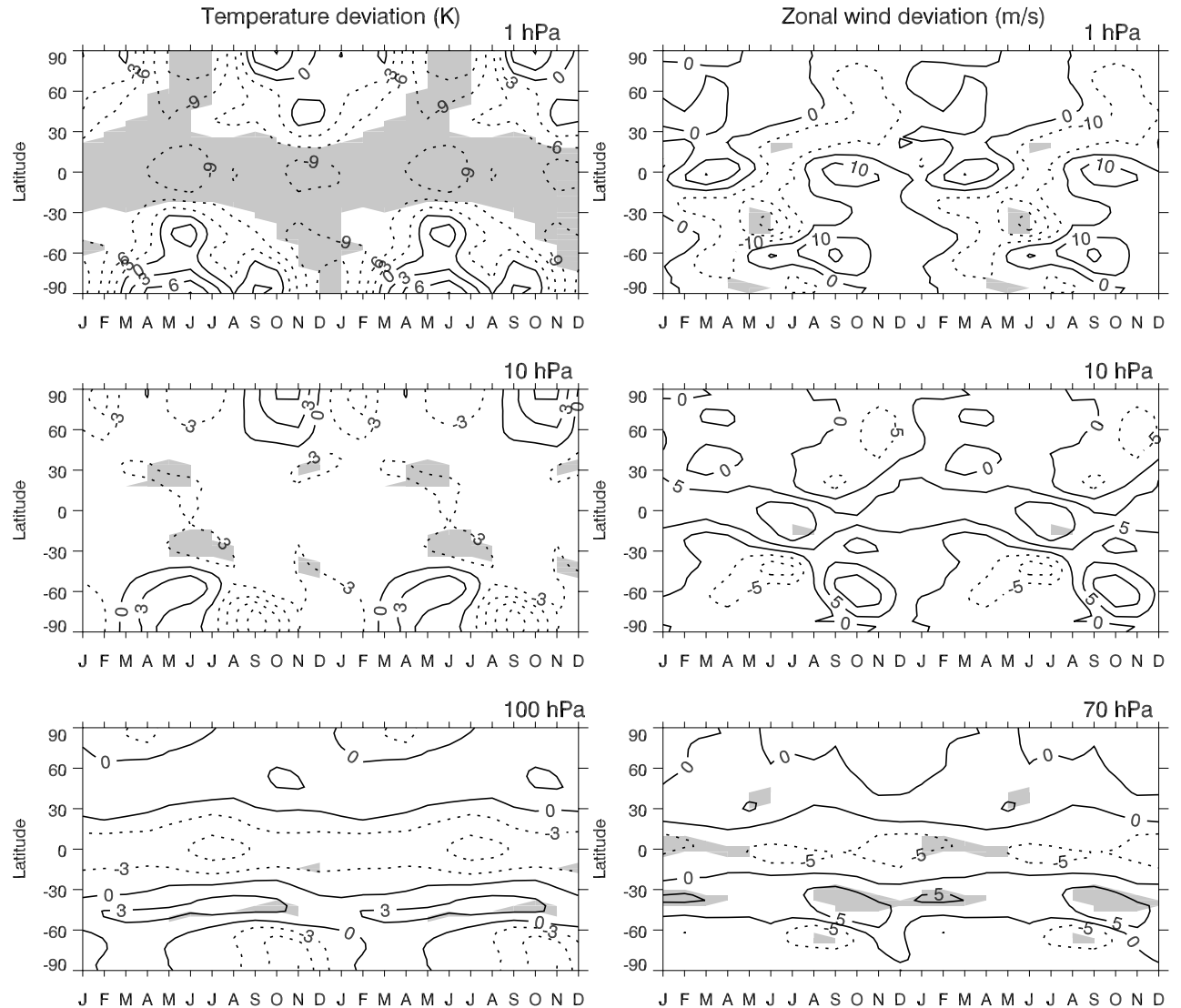

Fig. 5. Seasonal variation of the simulated temperature and zonal wind deviations from the observation data at 1,10 and $70 \mathrm{hPa}$. Shading marks "hotspots", the area where the deviations are significant. Twelve months of climatological data are repeated twice. 

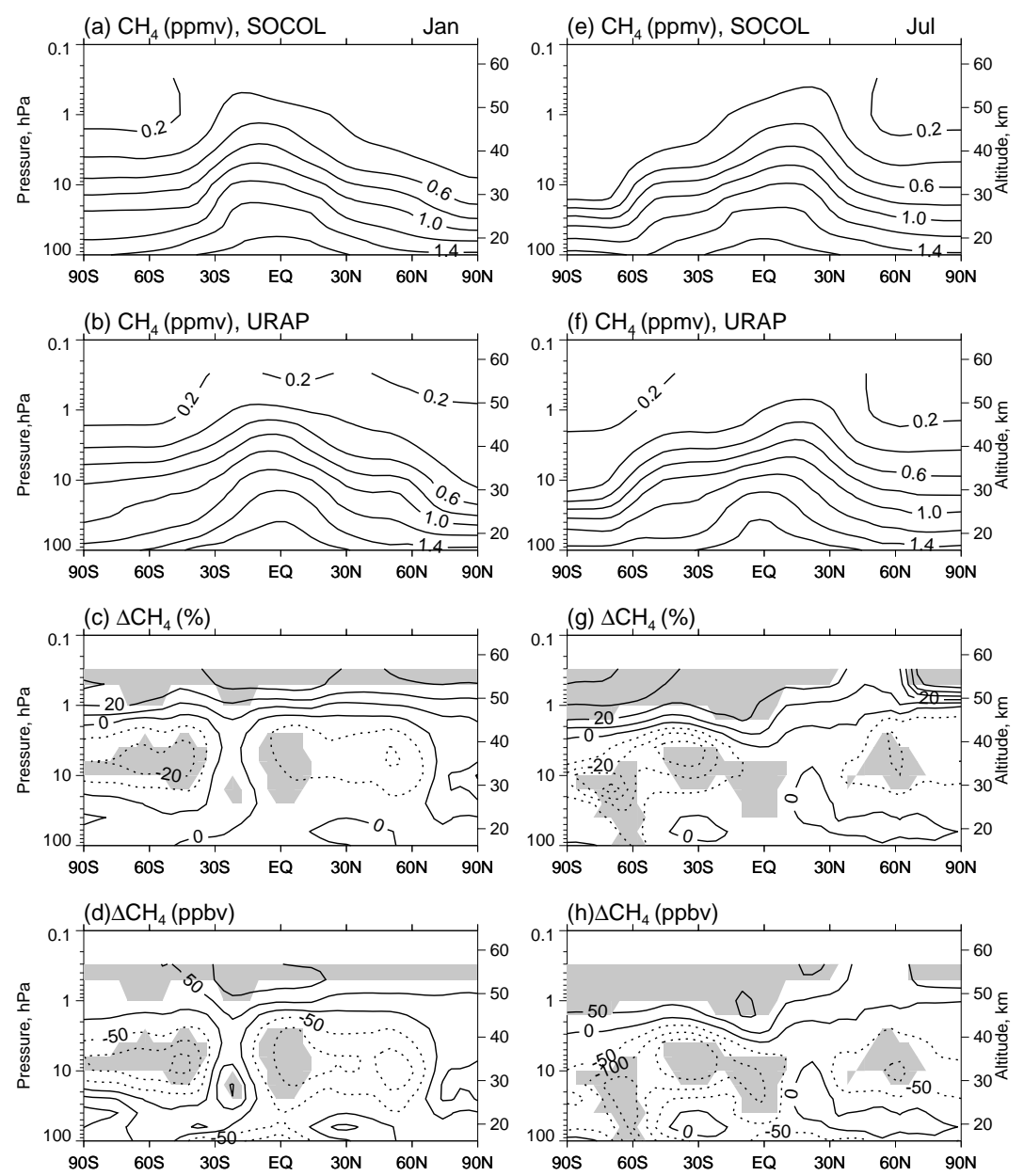

Fig. 6. Latitude-pressure cross-section of the $\mathrm{CH}_{4}$ (ppmv) for January (left panel) and July (right panel): simulated (a, b), observed (b, e), and their differences in steps of $\pm 1 \%(\mathbf{c}, \mathbf{d})$ and of $\pm 50 \mathrm{ppbv}(\mathbf{d}, \mathbf{h})$. Observed values are from URAP data set. Shading marks "hotspots", the area where the deviations are significant.

cold winter middle stratosphere, warm summer stratopause, and the polar temperature minimum associated with the formation of the polar vortices.

\subsubsection{Model/observation difference fields}

A simple visual comparison of temperature and zonal wind fields has often been used to validate CCMs (e.g., Takigawa et al., 1999; Hein et al., 2001). From this kind of comparison one can only conclude how well a model reproduces the main observed features of the zonal mean temperature and zonal mean zonal wind structures in general. However, differences between simulated and observed fields do exist and it is very helpful to use a more quantitative analysis of model deviations from observations as it has been presented by Rozanov et al. (2001) and Jonsson et al. (2002). Due to noticeable discrepancies among the available reanalysis data (e.g., SPARC, 2002) it is difficult to judge the model performance precisely and to give recommendations on how a model could be improved. To estimate the significance of the model deficiencies we use a monthly mean observed climatology of the zonal wind (see Figs. 3a, b) and temperature (see Figs. 4a, b) and the standard deviation of these quantities described in Sect. 3. From the results of the 40-year long SOCOL integration we have also calculated the climatology of the zonal wind (see Figs. 1a, b) and temperature (see Figs. 2a, b) and their standard deviations. Using these data sets we have calculated the difference between the simulated and observed climatology and estimated the significance of these deviations. The difference between the simulated and observed fields is defined as significant if it exceeds the sum of the standard deviations of the simulated and observed fields.

Figures $3 \mathrm{c}, \mathrm{d}$ and $4 \mathrm{c}, \mathrm{d}$ show ensemble mean monthly mean deviations of the model from the observational data in January and July for zonal means of zonal wind and temperature respectively. The gray spots mark the area where the model deviations from the observational data are significant. In the zonal wind field these spots appear in the region of the 

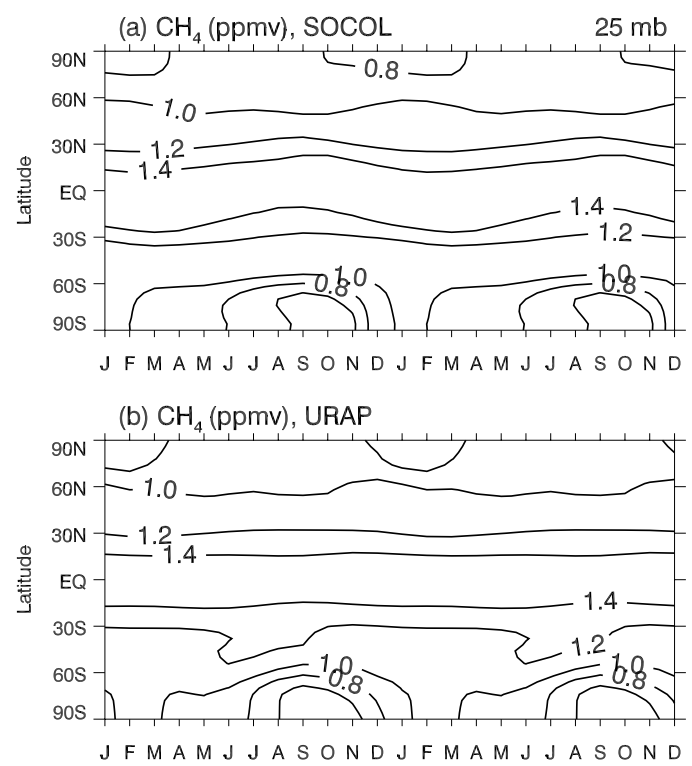

(c) $\triangle \mathrm{CH}_{4}(\%)$

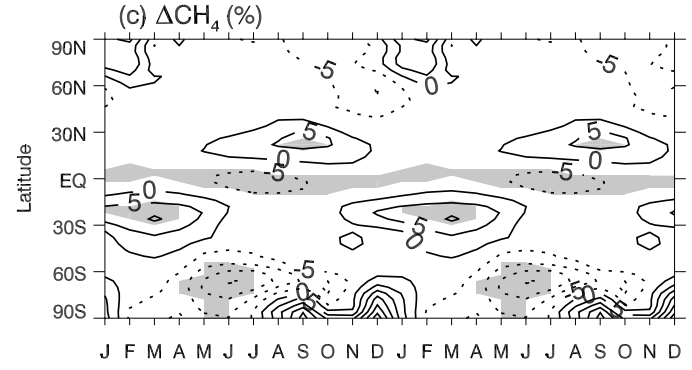

Fig. 7. Seasonal variations of the methane mixing ratio at $25 \mathrm{hPa}$ : (a) simulated, (b) observed, and (c) their difference in percent. Observed values are from the URAP data set. Shading marks "hotspots", the area where the deviations are significant.

extra tropical jets implying that SOCOL has a tendency to reproduce stronger (up to $5-10 \mathrm{~m} / \mathrm{s}$ ) jets in the upper troposphere. The marginally significant westerly bias $(5-10 \mathrm{~m} / \mathrm{s})$ around $10 \mathrm{hPa}$ and the weak easterly bias $(\sim 5 \mathrm{~m} / \mathrm{s})$ around $40 \mathrm{hPa}$ could be the result of the lack of QBO in the model and slight westerly QBO bias in the observation data. All other deviations appear to be insignificant. In the temperature field (Figs. 4c, d) the model substantially deviates from the observational data near the extra-tropical tropopause. The simulated temperature has a significant warm bias in the upper stratosphere in the tropics and in the summer hemisphere over high and middle latitudes. At the high latitude tropopause the discrepancies between simulated and observed data reach about $-6 \mathrm{~K}$ during winter and in the summer the deviation is up to $-10 \mathrm{~K}$. In the tropical lower stratosphere the simulated temperature has a cold bias of up to $-6 \mathrm{~K}$ and a weak positive bias just above this layer, which resembles the temperature anomalies due to the QBO (Giorgetta et al., 2004). The deviations are mostly negative, showing that the model is cold biased relative to the data.
Figure 5 shows the seasonal variation of the simulated temperature (left panel) and zonal wind (right panel) deviations from the observations at 1,10 and $70 \mathrm{hPa}$. The shaded areas mark the "hotspots", i.e. significant discrepancies between simulated and observed data. At $1 \mathrm{hPa}$ a significant cold bias of about $10 \mathrm{~K}$ has been found in December and January over the southern high latitudes, during all months in the tropical area, and over the high latitudes in the Northern Hemisphere (NH) in June and July. The positive deviations over the high latitudes in both hemispheres are not significant nor are most of the deviations of the zonal wind. At $10 \mathrm{hPa}$ the simulated temperature deviations from the observations are insignificant, except a small $(-3 \mathrm{~K})$ negative bias at $30^{\circ} \mathrm{S}$ and $30^{\circ} \mathrm{N}$. The simulated zonal wind at $10 \mathrm{hPa}$ deviates in the tropics during boreal summer, however, the deviations do not exceed $10 \mathrm{~ms}^{-1}$ and are only marginally significant. In the lower stratosphere the model has a cold bias at the equator, of up to $9 \mathrm{~K}$, and a warm bias of up to $3 \mathrm{~K}$ in the extra tropical area. The simulated zonal wind at $70 \mathrm{hPa}$ has an easterly bias in the tropical area and a westerly bias over the middle latitudes with a magnitude of $5 \mathrm{~ms}^{-1}$, however these biases are only marginally significant.

\subsubsection{Summary}

From the analysis of the zonal mean and seasonal variations of the zonal wind and temperature we conclude that during warm seasons our model does not have enough heating in the upper stratosphere at high latitudes and at the equator. This might be connected to the problem in the radiation code of MA-ECHAM4, which describes the absorption of solar UV radiation by ozone and oxygen with a rather simplified scheme. We will return to this problem in Sect. 5 . The temperature differences are most pronounced near the tropopause. These model deficiencies over high latitudes can be explained by the rough vertical model resolution in the upper troposphere-lower stratosphere (Roeckner et al., 2004). Some part of the cold bias in the tropical lower stratosphere can be explained by the absence of the QBO in the model (Giorgetta et al., 2004).

\section{Chemical aspects of the validation}

\subsection{Methane}

Altitude dependence. Methane is the most abundant hydrocarbon in the atmosphere and is useful as a tracer of atmospheric circulation because of its long photochemical lifetime ( $\sim 8-9$ years in the global atmosphere). Hence, the methane distribution is determined mainly by features of the circulation. Figure 6 shows the meridional cross section of the $\mathrm{CH}_{4}$ mixing ratio climatology simulated by SOCOL and observed by UARS together with their difference. The overall modeled zonal mean distribution $\left(\mathrm{CH}_{4}\right.$ decreases with height and latitude) resembles the observed one. As a result 

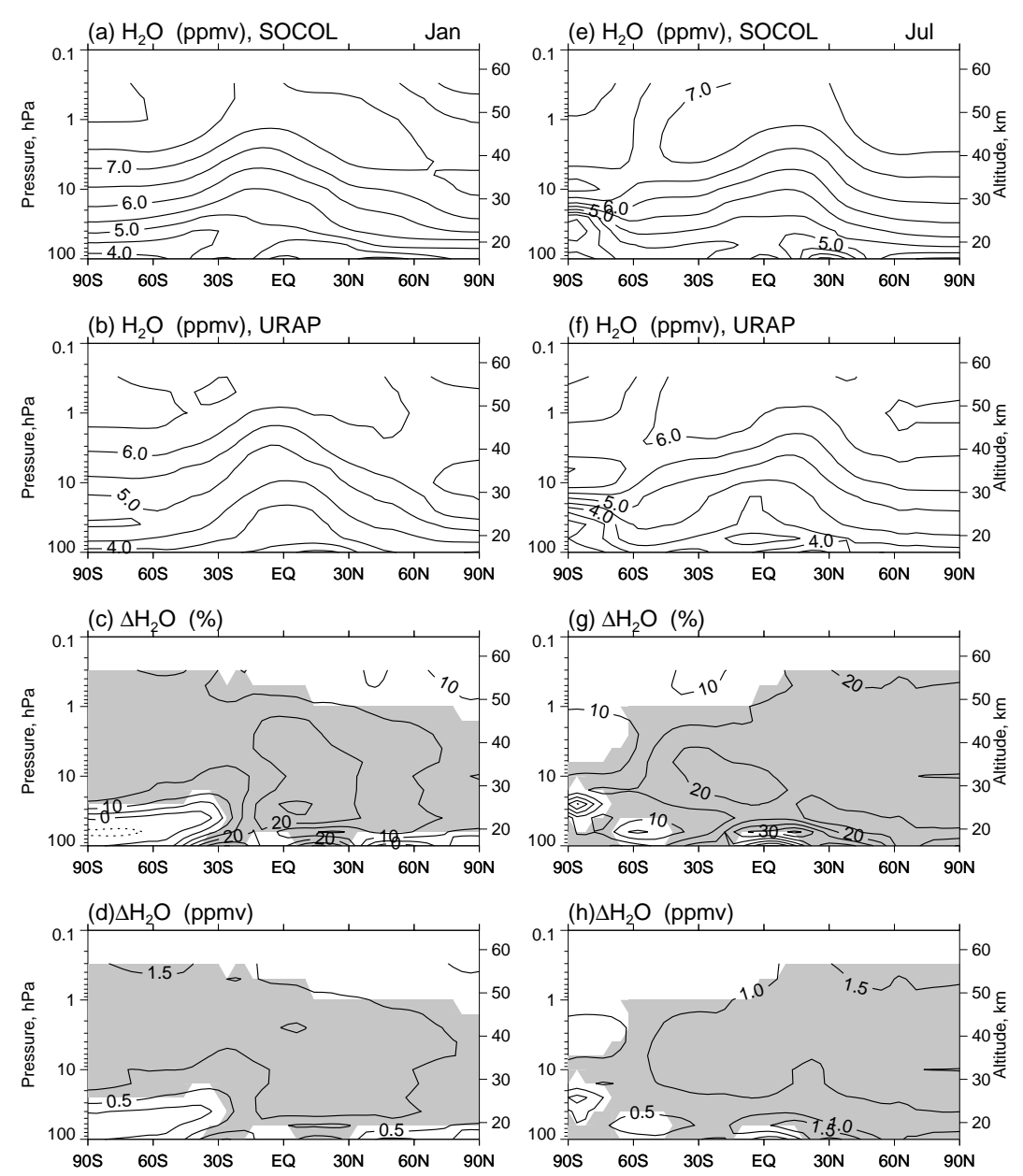

Fig. 8. Same as for Fig. 6, but for water vapor.

of transport by the Brewer-Dobson circulation, the tropical maxima of $\mathrm{CH}_{4}$ concentration is shifted to the North during boreal summer and to the South during boreal winter. The subtropical transport barriers are also reasonably simulated. The model significantly overestimates the methane mixing ratio in the mesosphere, which could be connected to the underestimation of chemical methane destruction by the hydroxyl or methane photolysis in Lyman- $\alpha$ line. Significant deviations of the simulated methane mixing ratio from the observations also take place in the middle stratosphere implying less intensive methane transport by the meridional circulation. The underestimation of the circulation intensity can be partially explained by the absence of the QBO in the model (Girogetta et al., 2004). The model reasonably reproduces the downward motion over the northern high latitudes in January, however in the Southern Hemisphere the polar vortex is too strong, leading to an underestimation of the $\mathrm{CH}_{4}$ mixing ratio at $10 \mathrm{hPa}$ by more then $20 \%(\sim 100 \mathrm{ppbv})$.

Seasonal cycle. Latitude-time variations in the observed and simulated zonal average mixing ratio of $\mathrm{CH}_{4}$ at $25 \mathrm{hPa}$ are shown in Fig. 7. The model reproduces the seasonal varia- tion over the middle and high latitudes, which is similar to the HALOE data with a relative minimum over the high latitudes during December-February for the NH and SeptemberNovember for the SH, while in the tropical area the simulated methane mixing ratio has a seasonal cycle, in contrast to the UARS data, which shows no apparent seasonal cycle. At $25 \mathrm{hPa}$, significant differences between simulated and observed data in the tropics are about $\pm 5 \%$ and in the southern high latitudes the difference reaches $-15 \%$ in May-June because of the downward transport of the air with low methane mixing ratio from the upper stratosphere starts earlier in the model than in the observations.

\subsubsection{Water vapor}

Altitude dependence. Water vapor is an important tracer in the upper troposphere and lower stratosphere. In both regions $\mathrm{H}_{2} \mathrm{O}$ is a source of $\mathrm{HO}_{\mathrm{x}}$ radicals, which are involved in photosmog reactions producing ozone in the upper troposphere and in catalytic ozone destruction cycles in the stratosphere. Figure 8 presents meridional cross-sections of water 


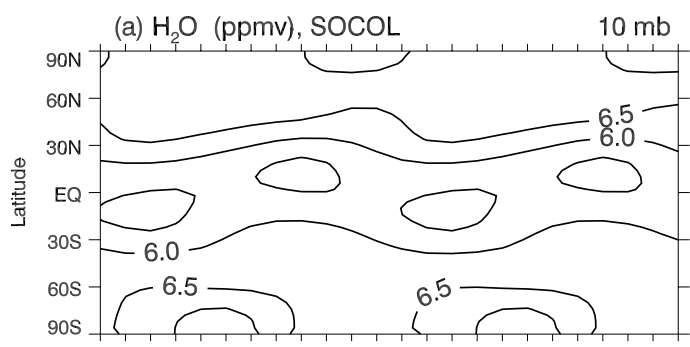

$J F M A M J J A S O N D J F M A M J J A S O N D$

(b) $\mathrm{H}_{2} \mathrm{O}$ (ppmv), URAP
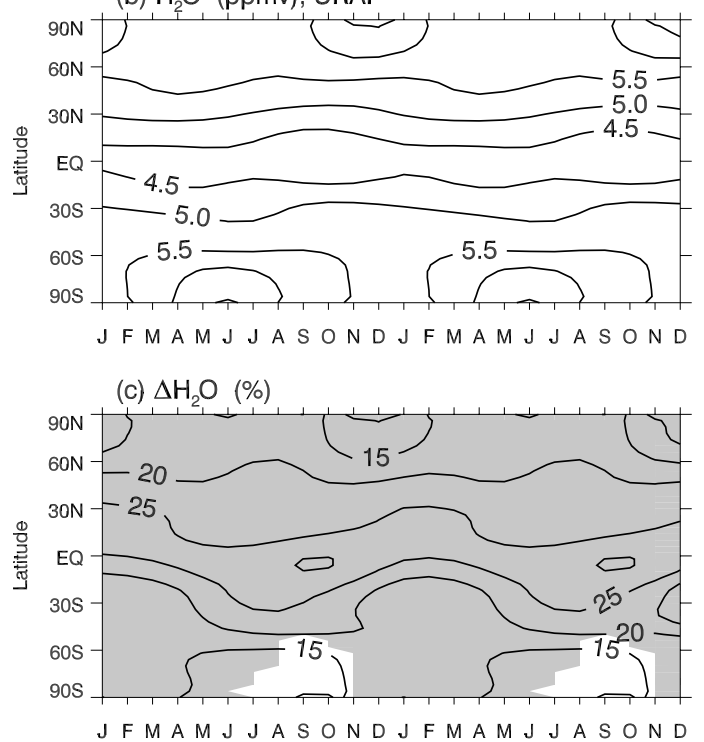

Fig. 9. Same as for Fig. 7 but for water vapor at $10 \mathrm{hPa}$.

vapor mixing ratios. The shape of the zonal-mean $\mathrm{H}_{2} \mathrm{O}$ distribution is well reproduced by SOCOL. However, the model overestimates mixing ratio of $\mathrm{H}_{2} \mathrm{O}$ compared to URAP data in the stratosphere by $0.5-1.0 \mathrm{ppmv}$ (or $10-20 \%$ ), which is within the range of accuracy of HALOE measurements (Harries et al., 1996). There are two sources of $\mathrm{H}_{2} \mathrm{O}$ in the stratosphere: $\mathrm{CH}_{4}$ oxidation in the stratosphere and upward transport of $\mathrm{H}_{2} \mathrm{O}$ from the troposphere. The latter depends in turn on the intensity of the upward branch of the BrewerDobson circulation, which determines vertical transport and on the $\mathrm{H}_{2} \mathrm{O}$ mixing ratio at the entry level. The $\mathrm{H}_{2} \mathrm{O}$ mixing ratio in the stratosphere is defined by chemical production from the methane oxidation cycle and by transport processes. Chemical destruction by reaction with exited oxygen plays a less important role. Transport processes tend to make stratospheric $\mathrm{H}_{2} \mathrm{O}$ closer to the $\mathrm{H}_{2} \mathrm{O}$ mixing ratio at the entry level, which is located in the tropical UTLS. Therefore, stronger circulation would usually lead to a decrease of the stratospheric $\mathrm{H}_{2} \mathrm{O}$. This means that in general, the overestimation problems of stratospheric $\mathrm{H}_{2} \mathrm{O}$ can be caused by: (i) overestimated chemical production, (ii) underestimated intensity of the meridional circulation; and (iii) overestimation of the $\mathrm{H}_{2} \mathrm{O}$ mixing ratio at the entry level. Figure 6
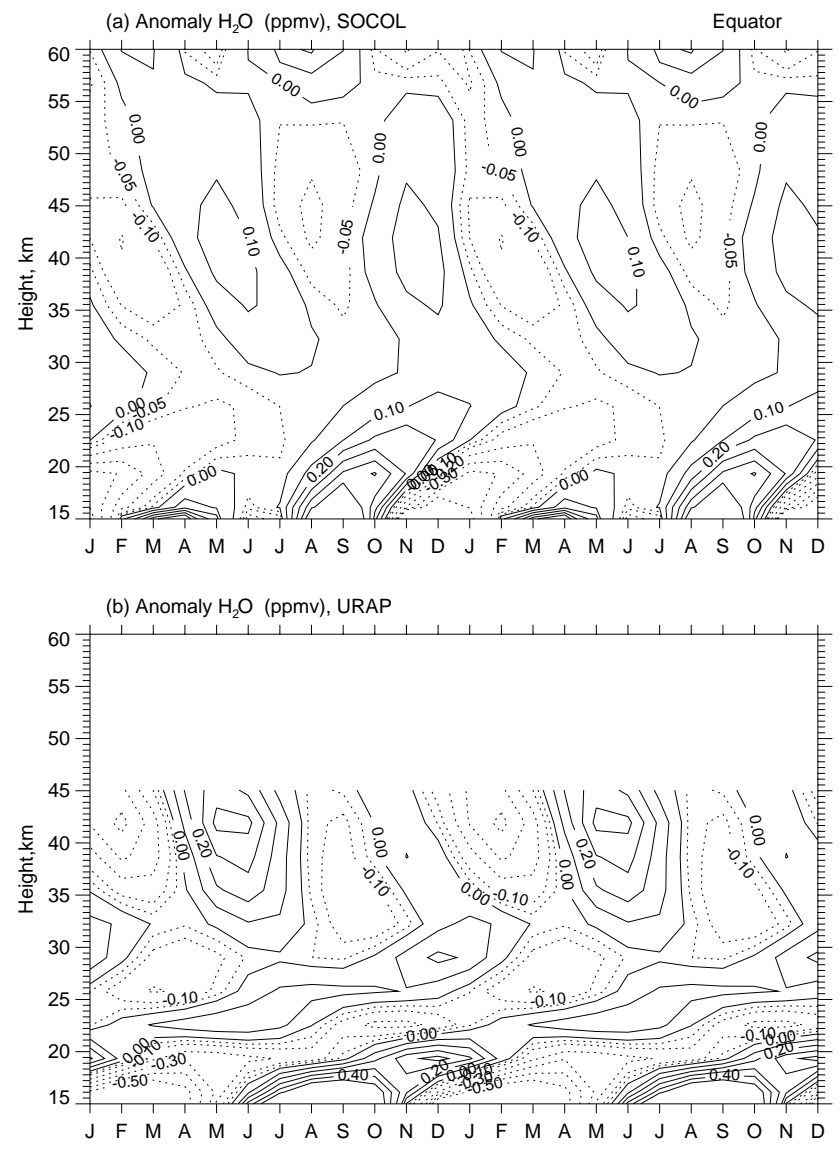

Fig. 10. Altitude-time evolution of water vapor mixing ratio over the equator, derived from (a) SOCOL simulation and (b) HALOE observation. Twelve months of climatological data are repeated twice. The negative (positive) contours are labeled in steps of 0.05 (0.1) ppmv.

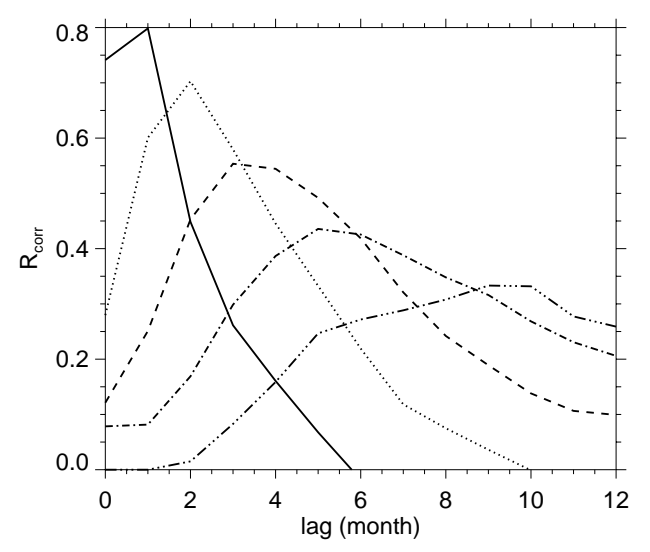

Fig. 11. Lagged correlation coefficients of deseasonalised $\mathrm{H}_{2} \mathrm{O}$ mixing ratio anomalies in the equatorial stratosphere at $16 \mathrm{~km}$ with the same quantity at 19.4 (solid line), 22.7 (dotted line), 25.9 (dashed line), 29.1 (dot-dashed line) and 32.3 (dot-dot-dashed line) $\mathrm{km}$ levels. 

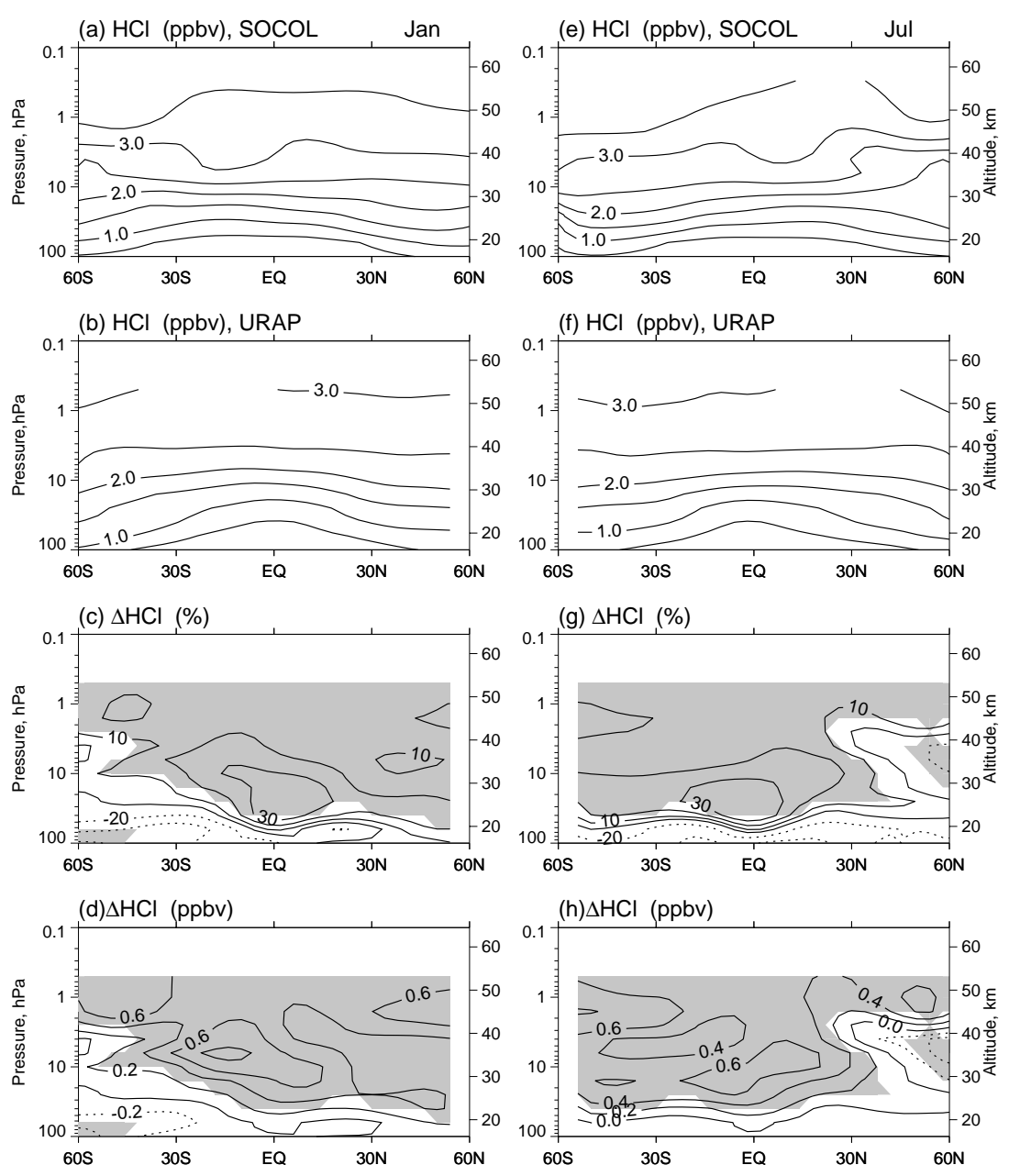

Fig. 12. Same as for Fig. 6 but for $\mathrm{HCl}$ (contour lines in steps of $0.5 \mathrm{ppbv}$ ). The difference in (c , g) is shown in steps of $\pm 10 \%$ and in (d, h) is shown in steps of $\pm 0.2 \mathrm{ppbv}$.

shows a small $(\sim 0.1 \mathrm{ppmv})$ deviation of $\mathrm{CH}_{4}$ mixing ratios in the middle stratosphere from the URAP data, which could explain only $\sim 0.2$ ppmv of the deviation of $\mathrm{H}_{2} \mathrm{O}$ mixing ratios. However, it is clear from Fig. 8 that the simulated $\mathrm{H}_{2} \mathrm{O}$ mixing ratio are overestimated by at least $1 \mathrm{ppmv}$, which implies that only the intensity of the meridional circulation or $\mathrm{H}_{2} \mathrm{O}$ mixing ratio at the entry level could be responsible. A slight underestimation of the simulated methane in comparison with URAP data implies that the intensity of the meridional transport in our model is underestimated, therefore the overestimation of stratospheric $\mathrm{H}_{2} \mathrm{O}$ could be connected to the slower meridional circulation or an overestimated $\mathrm{H}_{2} \mathrm{O}$ mixing ratio at the entry level. The later is more plausible explanation because the analysis of Figs. $8 \mathrm{~d}$, j shows that the deviation of the $\mathrm{H}_{2} \mathrm{O}$ mixing ratio in the stratosphere from URAP data is close to the deviation at the entry level.

Seasonal cycle. The seasonal variation of simulated $\mathrm{H}_{2} \mathrm{O}$ mixing ratios at $10 \mathrm{hPa}$ is compared with URAP data in Fig. 9. The model and URAP data do not show a sufficiently strong annual cycle in the tropical middle stratosphere. Over the high latitudes both model and observations reveal elevated $\mathrm{H}_{2} \mathrm{O}$ mixing ratios during wintertime, associated with aged air chemically depleted with respect to $\mathrm{CH}_{4}$, descending into the polar vortices.

Figure 10 presents a comparison of the simulated and URAP-derived data set of the altitude-time anomaly in the $\mathrm{H}_{2} \mathrm{O}$ mixing ratio (deviation from annual mean) over the equator. The model reproduces the vertical propagation of the dry (negative) and wet (positive) anomalies induced by the water vapor changes in the lower stratosphere, i.e. the water vapor "tape recorder" described by Mote et al. (1998). However, the model upward transport is up to twice as fast as observed. In order to quantitatively estimate the intensity of the upward water vapor transport we have calculated lagged correlations between deseasonalized $\mathrm{H}_{2} \mathrm{O}$ mixing ratio anomalies at $16 \mathrm{~km}$ altitude and at different altitudes in the equatorial stratosphere. The correlation coefficients are plotted in Fig. 11 for the 19.4, 22.7, 25.9, 29.1 and $32.3 \mathrm{~km}$ 

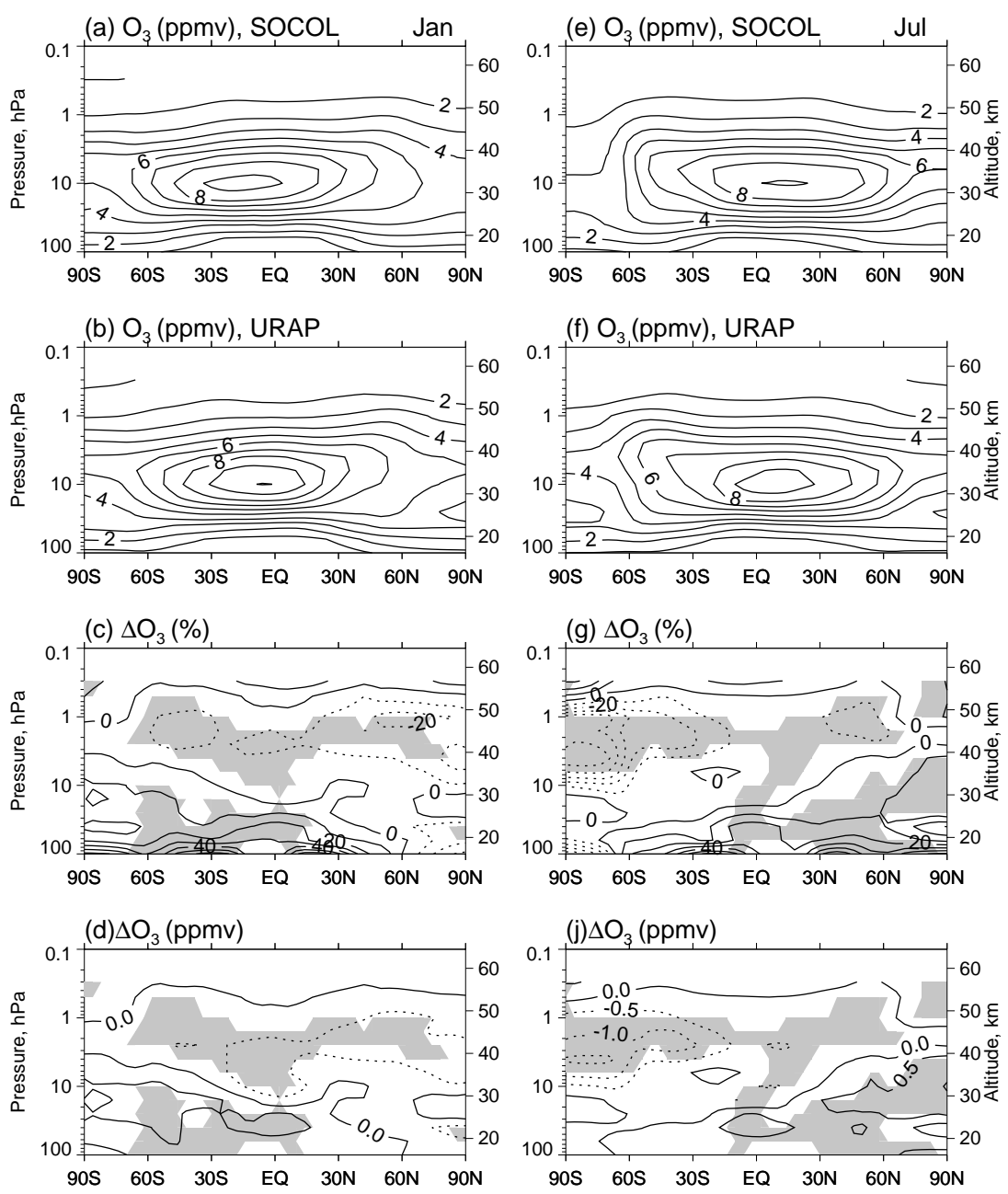

Fig. 13. Same as for Fig. 6 but for $\mathrm{O}_{3}$ (contour lines in steps of 1 ppmv). ). The difference in (c , g) is shown in steps of $\pm 10 \%$ and in (d, h) is shown in steps of $\pm 0.5 \mathrm{ppbv}$.

levels. The time when the maximum correlation is reached and the distance between levels allow the estimation of the vertical velocities in the equatorial lower stratosphere. For the plotted data the mean vertical velocity between 16 and $32.3 \mathrm{~km}$ is equal to $\sim 0.6 \mathrm{~mm} / \mathrm{s}$, which exceeds the value obtained from the observed $\mathrm{H}_{2} \mathrm{O}$ distribution by about 50 $90 \%$. The vertical velocity is larger in the lower stratosphere (around $1 \mathrm{~mm} / \mathrm{s}$ ), while between 29.1 and $32.3 \mathrm{~km}$ its magnitude is about $0.25 \mathrm{~mm} / \mathrm{s}$. Similar distributions of the vertical velocities have been reported by Steil et al. (2003). It is still not clear which part of the model is responsible for these discrepancies between the simulated and observed "tape recorder" features. In the upper stratosphere the model quantitatively matches the observed semi-annual oscillation with positive anomalies during the boreal summer (Fig. 10).

\subsection{2 $\mathrm{HCl}$}

$\mathrm{HCl}$ is a reservoir species for the chlorine group. Its mixing ratio in the upper stratosphere and mesosphere characterizes the level of the total reactive chlorine available for the chemistry. $\mathrm{HCl}$ plays an important role in the polar ozone chemistry, providing a source for active chlorine radicals through the heterogeneous chlorine activation process. The simulated distribution of $\mathrm{HCl}$ for January and July is presented in Fig. 12 in comparison with URAP data. The simulated $\mathrm{HCl}$ mixing ratio mimics some of the observed features. The $\mathrm{HCl}$ mixing ratio increases with altitude from $\sim 0.5 \mathrm{ppbv}$ in the lower stratosphere up to $3.5 \mathrm{ppbv}$ in the mesosphere. The model also captures the observed decrease of the $\mathrm{HCl}$ mixing ratio with latitude in the lower stratosphere and an uniform latitudinal distribution in the middle stratosphere. However, the observed latitudinal gradient is much steeper in the lower stratosphere than in the simulations. The overall agreement is within $\sim 20 \%$, but the error exceeds $30 \%$ in the tropical lower 

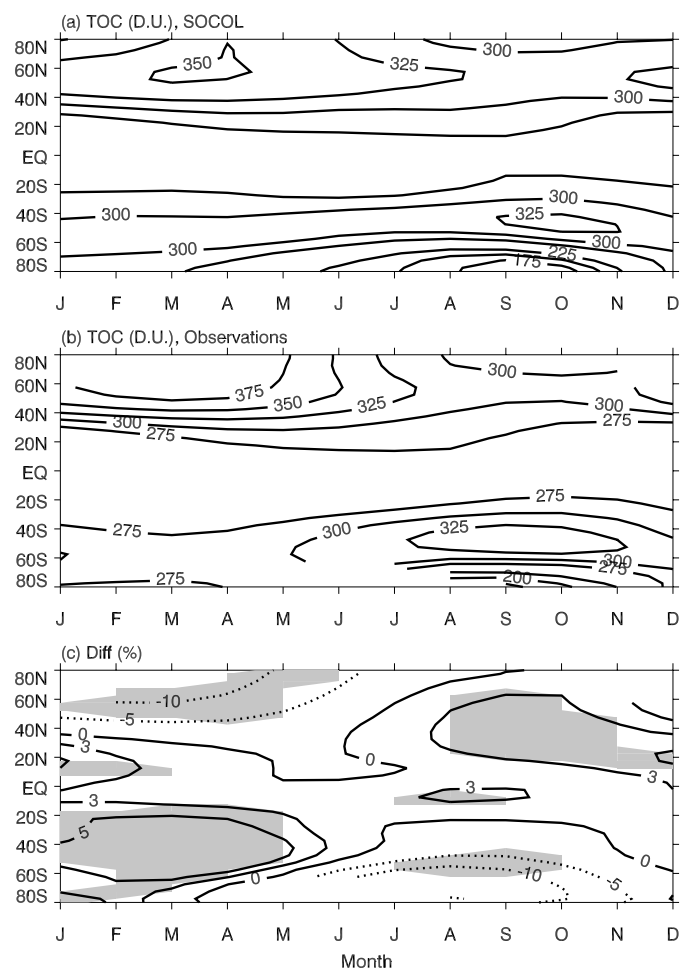

Fig. 14. Seasonal variation of the total ozone: (a) simulated, (b) observed, and (c) their difference in percents. The observed values are from TOMS/SBUV merged data set. Shading marks "hotspots", the area where the deviations are significant.

stratosphere where the $\mathrm{HCl}$ mixing ratio is much lower. The simulated $\mathrm{HCl}$ mixing ratio is systematically overestimated by the model, which can be related in part to the underestimated intensity of the meridional circulation and in part to the model run set-up. Here we analyze the results of the 40year model run performed with the mixing ratio of the source gases typical for the year 1995. Because of the 5-6 year time lag between stratospheric chlorine and the total chlorine in the near surface air, the stratospheric chlorine in our steadystate run are more representative of the late 90's . On the other hand, the URAP climatology of $\mathrm{HCl}$ in more representative of the earlier 90's, when the $\mathrm{HCl}$ mixing ratio should be to some extent lower than during the late 90 's.

\subsubsection{Ozone}

Altitude dependence. Figure 13 illustrates meridional cross sections of zonal mean monthly mean $\mathrm{O}_{3}$ mixing ratios, simulated by SOCOL and observed by UARS together with their difference. The distribution of the simulated ozone is in relatively good agreement with the observations throughout the stratosphere where the model errors remain basically within $\pm 10 \%$. The simulated maximum of the zonal mean ( $\sim 9$ ppmv) appears at the equator, at around $10 \mathrm{hPa}$, which is consistent with the observations. The so-called "banana" shape of the ozone distribution is also well captured by the model with high ozone regions extending to the upper polar stratosphere. The model significantly underestimates ozone over the southern high latitudes in the upper stratosphere during the austral winter season. The cause of the ozone underestimation could be in part related to (or identical to) the causes as for the underestimation of methane and the overestimation of water vapor in the same region: this could stem from a too strong isolation of the southern polar vortex and a too strong downward transport in the model. The fact that methane is longer-lived than ozone in these regions could be the reason for the methane discrepancy appearing only at lower altitudes. An underestimation of ozone in the upper stratosphere could in part explained by the overall overestimation of the reactive chlorine mentioned in Sect. 4.2.3, which leads to more intensive ozone destruction. The deviation of simulated ozone from the observation cannot explain the significant cold temperature bias near the stratopause (shown in Fig. 4). From our 1-D model (Rozanov et al., $2002 b$ ) we estimate that even $-10 \%$ ozone deficit could provide a cooling of only about $1.5 \mathrm{~K}$. A small positive bias in the tropical lower stratosphere around $40 \mathrm{hPa}$ and a negative bias at $10 \mathrm{hPa}$ can be explained by the absence of the QBO in the model (Giorgetta et al., 2004).

Seasonal cycle. The comparison of the seasonal variation of the simulated total ozone column (TOC) with the observations is presented in Fig. 14. SOCOL reproduces a seasonal maximum in the $\mathrm{NH}$ and a maximum and minimum in the SH with reasonable accuracy. The overall agreement between the model and the observation data composite is within $\pm 5 \%$ in the tropics, $\pm 10 \%$ in the northern middle and high latitudes, and within $\pm 10-20 \%$ in the southern middle and high latitudes. Significant deviations of the simulated total ozone from the TOMS climatology occur mostly from January to May and consists of an underestimation of the total ozone over the northern high latitudes and overestimation over the middle southern latitudes. This redistribution of the total ozone can be also explained by the above-mentioned slower-than-observed meridional circulation in the model. A similar pattern of the total ozone deviation appears again during austral winter and spring, however the total ozone deviations over the southern high latitudes are not significant, implying a good performance of the model in the area of the ozone "hole". Figure 15 illustrates the total ozone simulated by SOCOL in March over the NH and in October over the SH in comparison with corresponding satellite observations. The position and magnitude of the ozone "hole" is very well reproduced by SOCOL, confirming that the amount of PSCs during the spring season and chemical ozone destruction are reasonably well captured by the chemical routine of the model. The position of the total ozone maximum in the Australian sector is also well captured by SOCOL. The magnitude of the maximum is slightly underestimated, but this deviation is not significant. Some CCMs (see Austin et al., 2003, their Fig. 2) substantially overestimate the magnitude 
a) March, SOCOL

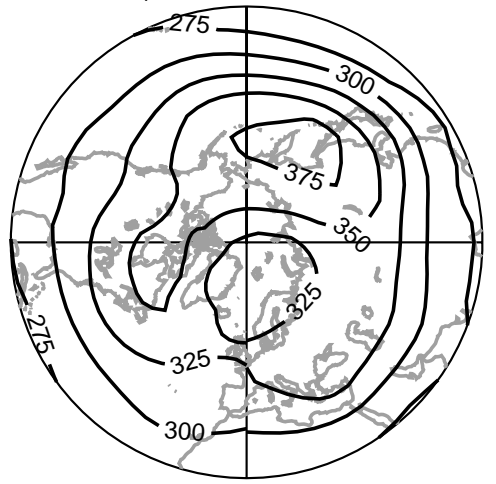

c) March, observation

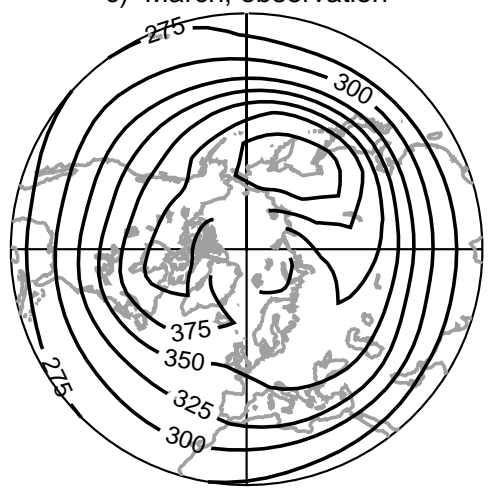

e) March, Diff (\%)

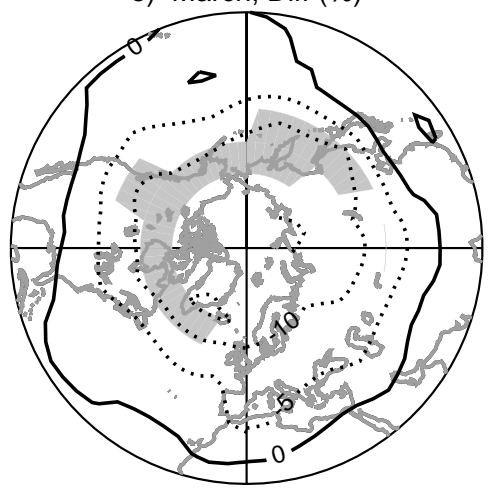

b) October, SOCOL

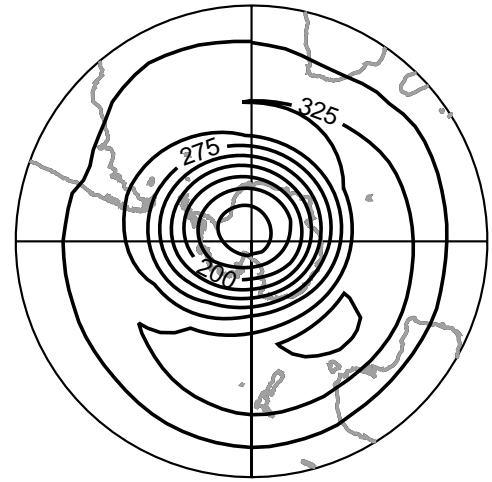

d) October, observation

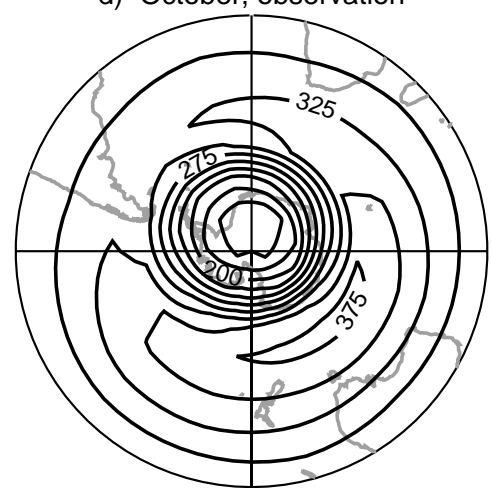

f) October, Diff (\%)

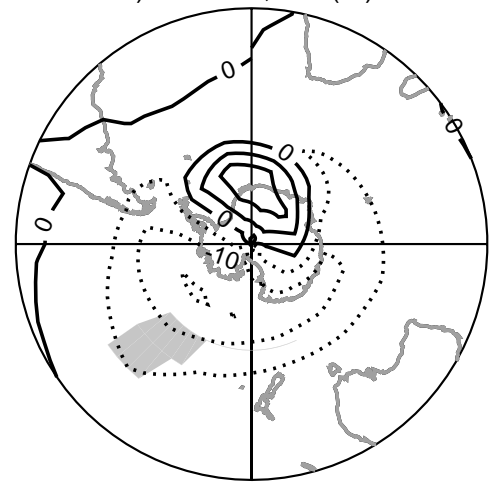

Fig. 15. Geographical distribution of the simulated $(\mathbf{a}, \mathbf{b})$ and observed $(\mathbf{c}, \mathbf{d})$ total ozone for March over the Northern Hemisphere and October over the Southern Hemisphere in Dobson Units (DU) and their difference (e, f) in percents. The observed values are from TOMS/SBUV merged data set. Shading marks "hotspots", the area where the deviations are significant.

of the total ozone maxima over the middle latitudes in the Australian sector. This could imply that the relevant wave forcing and subsequently meridional transport in these models are too strong, but SOCOL seems not to suffer from this problem. In March over the Northern Hemisphere, however, the underestimation of the simulated total ozone is more pronounced (up to 15\%) and significant over Pacific and North American sectors. As was mentioned before it could be a result of a weak meridional circulation in the model.

We have compared the pattern correlation and absolute deviation (not shown) of area weighted total ozone simulated by SOCOL and simulated by CCMs that participated in the model intercomparison presented by (Austin et al., 2003). The comparison shows that among the other models SOCOL has the smallest absolute deviation from the observations in the $\mathrm{SH}$, which is $\sim 5 \%$ and very high pattern correlation (more than 0.95) over both hemispheres. Over the Northern Hemisphere SOCOL underestimates the total ozone maximum in March by about 50 DU, but nevertheless has one of the smallest absolute deviations $(\sim 5 \%)$ of total area weighted ozone over the $\mathrm{NH}$ from the observational data among the models compared by Austin et al. (2003). 

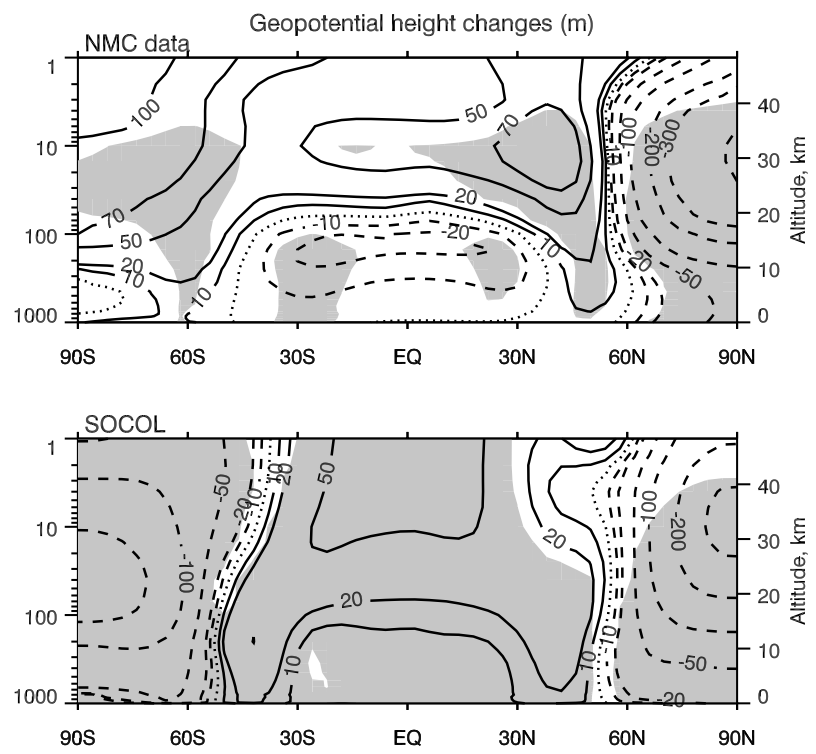

Fig. 16. Observed and simulated differences between positive and negative AO phase in the zonal mean geopotential heights (m). Shading marks the area where the difference is statistically significant at $90 \%$ confidence level.
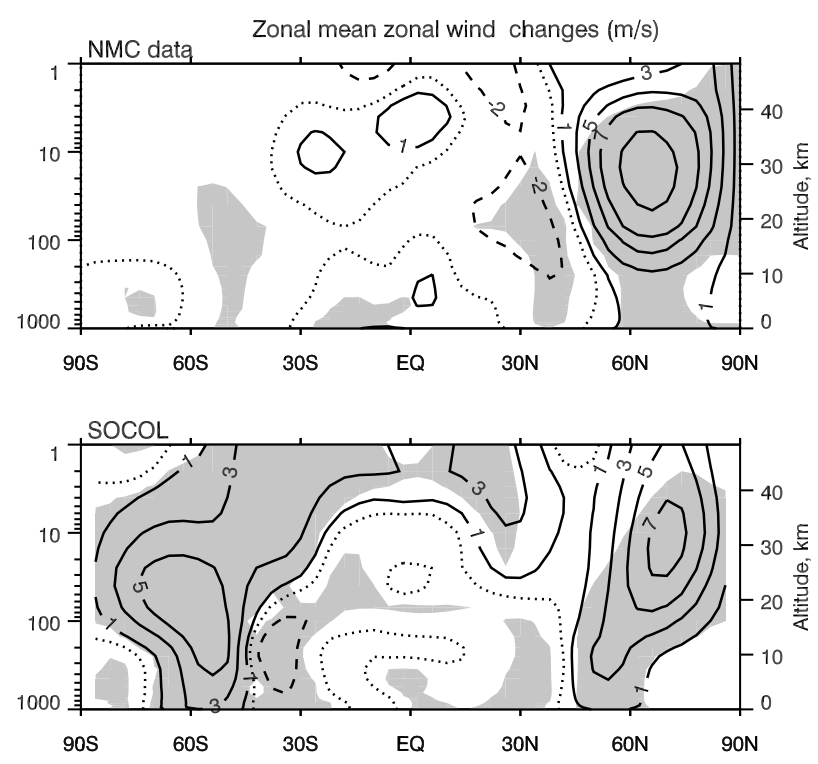

Fig. 17. Observed and simulated differences between positive and negative AO phase in the zonal mean zonal wind $\left(\mathrm{ms}^{-1}\right)$. Shading marks the area where the difference is statistically significant at $90 \%$ confidence level.

5.2 Sensitivity of ozone and temperature to the strength of the Arctic winter vortex

As was mentioned in Sect. 3 we divided the simulated and observed data into two groups according to the phase of Arctic Oscillation during the boreal winter season to
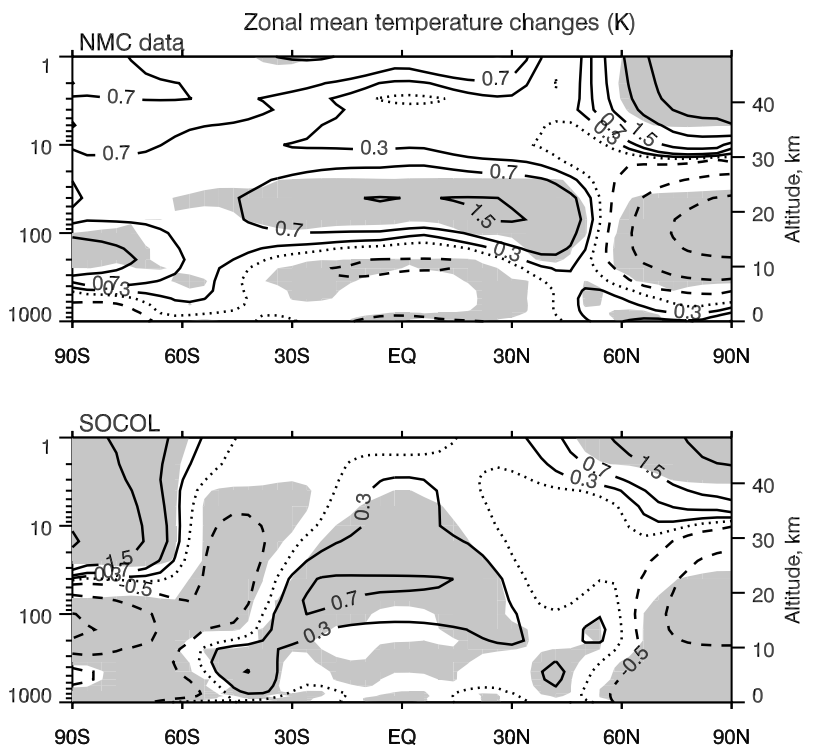

Fig. 18. Observed and simulated differences between positive and negative AO phase in the zonal mean temperature (K). Shading marks the area where the difference is statistically significant at $90 \%$ confidence level.
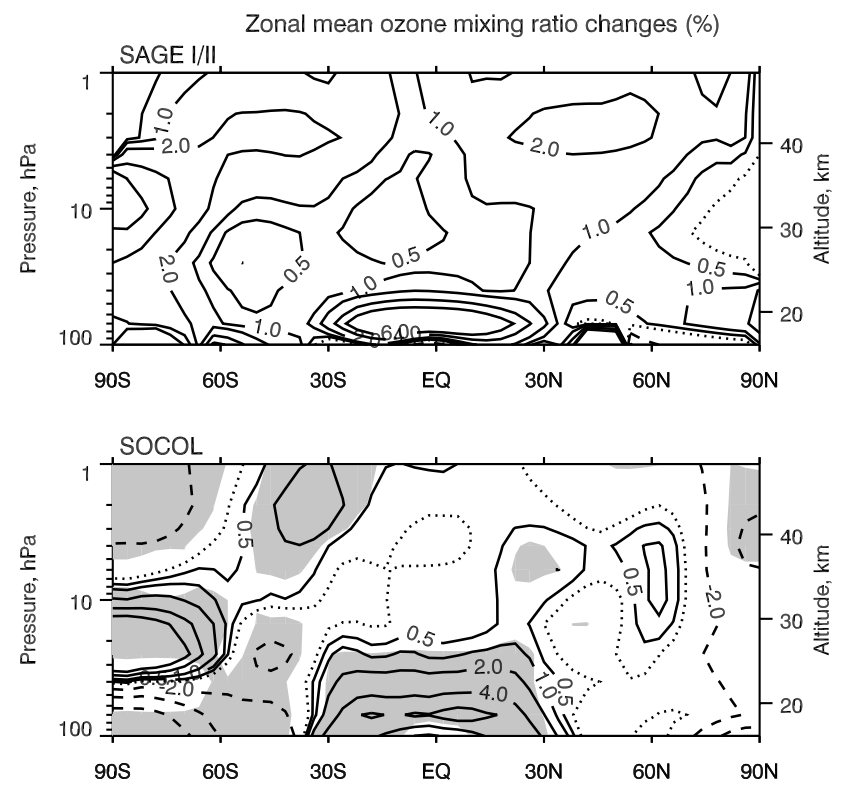

Fig. 19. Observed and simulated differences between positive and negative AO phase in the zonal mean ozone mixing ratio (\%).Shading marks the area where the difference is statistically significant at $90 \%$ confidence level.

analyze the response of the atmosphere to the strength of the polar vortex. Figures 16-19 illustrate the differences between these two composites in zonal mean geopotential height, zonal wind, temperature and ozone mixing ratio averaged over the boreal winter season (December-JanuaryFebruary). Figure 16 shows that the simulated and observed 
differences in geopotential heights are broadly similar in the $\mathrm{NH}$ and tropical stratosphere. The deepening of the polar vortex and formation of the ridges over mid-latitudes for the positive AO phase is clearly visible and statistically significant in both data sets. The zonal wind difference in the composite (Fig. 17) consists of an acceleration of the PNJ by $7-10 \mathrm{~ms}^{-1}$ in the simulated and observed data. Figure 18 demonstrates the pattern of the temperature response. The simulated and observed temperature responses over the $\mathrm{NH}$ are similar and consist of a pronounced dipole-like structure with cooling (warming) in the middle-lower (upper) stratosphere. In the tropics, the model matches the observed warming in the lower stratosphere, although the magnitude of the simulated warming is about 2 times smaller. The simulated and observed temperature changes in the upper tropical stratosphere are not statistically significant. The ozone response is shown in Fig. 19. The simulated and observed changes are in qualitative agreement only in the lower tropical stratosphere, where the model simulates a statistically significant increase the ozone mixing ratio. This theoretically expected feature is also visible in the observation data, however it does not appear to be statistically significant. In the model the warming in the tropical stratosphere and subsequent decrease of the equator-pole temperature gradient in the Southern Hemisphere leads to the westerly acceleration of the zonal wind in the stratosphere over middle latitudes, formation of the dipole-like structure and negative anomalies in the geopotential heights over the high latitudes. However, none of these features can be seen in the observation data, therefore it is hard to say how real they are.

\section{Conclusions}

In this paper we presented a description of a new modeling tool, the CCM SOCOL, together with the validation of the simulated present-day climatology against a variety of observational data. We also present an example of processesoriented validation. While the model performance is quite satisfactory based on an overall inspection of simulated fields and on a proper statistical analysis, we have identified a number of weaknesses that need to be addressed for the future improvement of the model. In particular, the analysis of the simulated zonal wind and temperature deviations shows that for an improvement it will be necessary to pay special attention to the tropopause region at high latitudes as well as to the description of the processes in the upper stratosphere and mesosphere, where significant cold biases have been found in the model during boreal summer.

The model's too cold upper stratosphere is most likely related to the radiation code of MA-ECHAM4 (see Sect. 4), which does not take into account the absorption of solar irradiance for wavelengths shorter than $250 \mathrm{~nm}$. To illustrate the importance of this spectral region we have applied a 1$\mathrm{D}$ radiative convective model (RCM) described by Rozanov

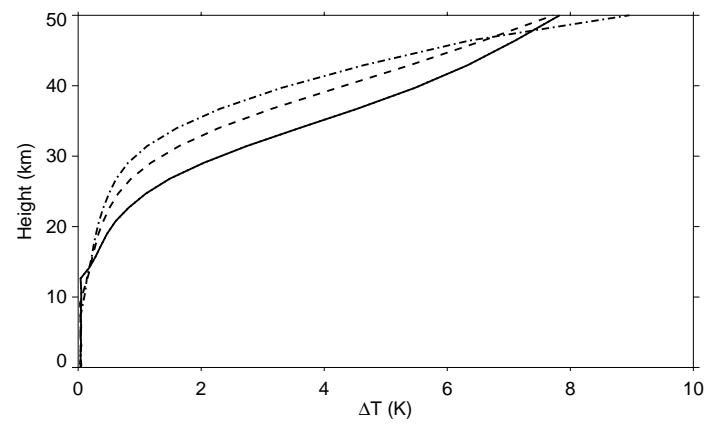

Fig. 20. Temperature difference due to absorption of the solar irradiance in the $120-250 \mathrm{~nm}$ spectral interval calculated with 1-D $\mathrm{RCM}$ for three cases: Tropical atmosphere model, $\mathrm{SZA}=45^{\circ}$, duration of the day $=12 \mathrm{~h}$ (solid line); Middle latitude summer atmosphere model, $\mathrm{SZA}=60^{\circ}$, duration of the day $=14.4 \mathrm{~h}$ (dashed line); Subarctic summer atmosphere model, $\mathrm{SZA}=70^{\circ}$, duration of the day $=24 \mathrm{~h}$ (dash-dotted line).

et al. (2002b) and calculated the temperature profiles with and without absorption of the solar irradiance in the spectral region $120-250 \mathrm{~nm}$. Temperature differences due to absorption of the solar irradiance in the $120-250 \mathrm{~nm}$ spectral interval have been calculated with the 1-D RCM for three cases: (1) a tropical atmosphere model, with Solar Zenith Angle (SZA) $=45^{\circ}$, duration of the day $(\mathrm{DoD})=12 \mathrm{~h}$; (2) a middle latitude summer atmosphere model, with $\mathrm{SZA}=60^{\circ}$, $\mathrm{DoD}=14.4 \mathrm{~h}$; (3) a subarctic summer atmosphere model, with $\mathrm{SZA}=70^{\circ}, \mathrm{DoD}=24 \mathrm{~h}$. The results are depicted in Fig. 20, suggesting that near the stratopause the contribution of the $120-250 \mathrm{~nm}$ spectral region could reach up to $9 \mathrm{~K}$. Therefore we hypothesize that the missing source of heat would substantially improve temperature and zonal wind distributions in the summer extra-tropical upper stratosphere and mesosphere also in the 3-D model.

The simulated descent of air is too strong in the polar stratosphere, leading to a significant underestimation of $\mathrm{CH}_{4}$ and $\mathrm{O}_{3}$ mixing ratios in this area. An analysis of the water vapor zonal mean and seasonal distributions reveals an overestimation of stratospheric $\mathrm{H}_{2} \mathrm{O}$, which is probably related to the transport of $\mathrm{H}_{2} \mathrm{O}$ from the troposphere. The analysis of the total ozone and some other quantities suggests that the simulated meridional circulation is too weak. For the further improvement of the model the parameterization of the unresolved wave forcing should be reconsidered to provide stronger wave drag and more intensive meridional circulation. The simulated distribution of the temperature, ozone and source gases in the tropical stratosphere can be also improved if the model is capable of reproducing the QBO.

As a process-oriented part of the validation we analyzed how SOCOL reproduces the relationship between the phase of $\mathrm{AO}$ and temperature and ozone fields. During the boreal winter (DJF) a signature of the positive AO phase or strong northern polar vortex is clearly visible in the observed and 
simulated data. Therefore the applied approach can be used for the validation of CCMs. SOCOL reasonably well reproduces AO-like patterns of the inter-annual variability, which consist of a deepening of the polar vortex and an acceleration of the PNJ during positive AO phases. The model also captures the concomitant deceleration of the meridional circulation, the subsequent warming, and the ozone increase in the lower tropical stratosphere. The model matches the pronounced dipole-like temperature response over the northern high-latitudes. However, the simulated warming in the tropical lower stratosphere is underestimated by a factor of 2 . The simulated ozone increase in the tropical lower stratosphere for the positive AO phase is in general agreement with observation, however the observed ozone response is not statistically significant. Additional observation and simulation data should be analyzed in order to elucidate the causes of the noticeable disagreement between simulated and observed atmospheric imprints of the $\mathrm{AO}$ phase.

Despite these model deficiencies, the overall performance of the modeling tool SOCOL is reasonable and many features of the real atmosphere are simulated rather well. The SOCOL has been ported for regular PCs and shows good wall-clock performance. Thus, many research groups can use it for studies of chemistry-climate problems even without access to large super-computer facilities.

\section{Software availability}

- Name of the software: Modeling tool for studies Solar Climate Ozone links (SOCOL)

- Contact address: PMOD/WRC, Dorfstrasse, 33, CH-7260, Davos Dorf, Switzerland

- Telephone and fax: tel. +41081 4175138, fax. +41 081 4175100

- E-mail: t.egorova@pmodwrc.ch

- Hardware required: Intel Pentium based PC, 512 MB memory at least Software required: LINUX, Fujitsu/Lahey FORTRAN

- Availability and cost: signed Software License Agreement available from the authors, appropriate citation required, collaboration preferable, free of charge.

Acknowledgements. This paper is based upon work supported by the by the Swiss Federal Institute of Technology, Zürich and PMOD/WRC, Davos, Switzerland. The work of V. Zubov was supported by INTAS (grant INTAS-01-0432) and RFFI (grant 02-05-65399). We thank the SPARC, URAP and TOMS Data Centers for providing the data and C. Hoyle and P. Forney for editing the manuscript. ECMWF ERA-40 data used in this study have been obtained from the ECMWF data server

Edited by: M. Dameris

\section{References}

Austin, J., Shindell, D., Beagley, S. R., et al.: Uncertainties and assessments of chemistry-climate models of the stratosphere, Atmos. Chem. Phys., 3, 1-27, 2003,

SRef-ID: 1680-7324/acp/2003-3-1.

Brönnimann, S., Luterbacher, J., Staehelin, J., and Svendby, T.: An extreme anomaly in stratospheric ozone over Europe in 1940-1942, Geophys. Res. Lett., 31, L08101, doi:10.1029/2004GL019611, 2004.

Butchart, N. and Austin, J.: On the relationship between the quasibiennial oscillation, total chlorine and the severity of the Antarctic ozone hole, Q. J. R. Meteorol. Soc., 122, 183-217, 1996.

Butchart, N. and Austin, J.: Middle Atmosphere Climatologies from the Troposphere-Stratosphere Configuration of the UKMO's Unified Model, J. Atmos. Sci., 55, 2782-2809, 1998.

Carslaw, K. S., Luo, B. P., and Peter, Th.: An analytic expression for the composition of aqueous $\mathrm{HNO}_{3}-\mathrm{H}_{2} \mathrm{SO}_{4}$ stratospheric aerosols including gas phase removal of $\mathrm{HNO}_{3}$, Geophys. Res. Lett., 22, 1877-1880, 1995.

Charron, M. and Manzini, E.: Gravity waves from fronts: Parameterization and middle atmosphere response in a general circulation model, J. Atmos. Sci., 59, 923-941, 2002.

DeMore, W. B., Sander, S. P., Golden, D. M., Hampson, R. F., Kurylo, M. J., Howard, C. J., Ravishankara, A. R., Kolb, C. E., and Molina, M. J.: Chemical Kinetics and Photochemical Data for Use in Stratospheric Modeling, Evaluation 12, JPL Publication, 97-4, 1997.

Egorova, T. A., Rozanov, E. V., Schlesinger, M. E., Andronova, N. G., Malyshev, S. L., Zubov, V. A., and Karol, I. L.: Assessment of the effect of the Montreal Protocol on atmospheric ozone, Geoph. Res. Lett., 28, 2389-2392, 2001.

Egorova, T. A., Rozanov, E. V., Zubov, V. A., and Karol, I. L.: Model for Investigating Ozone Trends (MEZON), Izvestiya, Atmospheric and Oceanic Physics, 39, 277-292, 2003.

Egorova, T., Rozanov, E., Manzini, E., Haberreiter, M., Schmutz, W., Zubov, V., and Peter, T.: Chemical and dynamical response to the 11-year variability of the solar irradiance simulated with a chemistry-climate model, Geophys. Res. Lett., 31, L06119, doi:10.1029/2003GL019294, 2004.

Eyring, V., Harris, N., Rex, M., et al.: Comprehensive Summary on the Workshop on "Process-Oriented Validation of Coupled Chemistry-Climate Models", SPARC, Newsletter, 23, 5-11, 2004.

Fouquart, Y. and Bonnel, B.: Computations of solar heating of the Earth's atmosphere: A new parameterization, Beitr. Phys. Atmos., 53, 35-62, 1980.

Giorgetta, M., B. Steil, C. Brühl, and E. Manzini: The role of the QBO in the climate and chemistry of the stratosphere in a transient simulation of 1960 to 2000, poster presented at 3rd General Assembly of SPARC, Victoria, Canada, 2004.

Gleckler, P. E.: AMIP Newsletter: AMIP-II guidelines, Lawrence Livermore Natl. Lab, Livermore, Calif., 1996.

Hanson, D. and Maursberger, K.: Laboratory studies of the nitric acid trihydrate: Implications for the south polar stratosphere, Geophys. Res. Lett., 15, 855-858, 1988.

Harries, J. E., Russell III, J. M., Tuck, A. F., et al.: Validation of measurements of water vapor from the halogen occultation experiment (HALOE), J. Geophys. Res., 101 (D6), 10 205-10 216, 1996. 
Hein, R., Dameris, M., Schnadt, C., et al.: Results of an interactively coupled atmospheric chemistry - general circulation model: Comparison with observations, Ann. Geophys., 19, 435457, 2001,

SRef-ID: 1432-0576/ag/2001-19-435.

Hines, C. O.: Doppler spread parameterization of gravity wave momentum deposition in the middle atmosphere, 1, Basic formulation, J. Atmos. Solar Terr. Phys., 59, 371-386, 1997 a.

Hines, C. O.: Doppler spread parameterization of gravity wave momentum deposition in the middle atmosphere, 2, Broad and quasi monochromatic spectra and implementation, J. Atmos. Solar Terr. Phys., 59, 387-400, 1997b.

Intergovernmental Panel of Climate Change: Climate Change 2001: The Scientific Basis, Cambridge Univ. Press, New York, 881, 2001.

Jacobson, M. Z. and Turco, R. P.: SMVGEAR: A sparsematrix, vectorized code for atmospheric models, Atmos. Environ., 28 273-29 284, 1994.

Jonsson, A., de Grandpre, J., and McConnell, J. C.: A comparison of mesospheric temperatures from the Canadian Middle Atmospheric Model and HALOE observations: Zonal mean and signature of the solar diurnal tide, Geophys. Res. Lett., 29, doi:10.1029/2001GL014476, 2002.

Kodera, K. and Kuroda, Y.: Dynamical response to the solar cycle, J. Geophys. Res., 107 (D24), 4749, doi:10.1029/2002JD002224, 2002.

Manzini, E. and McFarlane, N. A.: The effect of varying the source spectrum of a gravity wave parameterization in the middle atmosphere general circulation model, J. Geophys. Res., 103, 31523 $31539,1998$.

Manzini, E., McFarlane, N. A., and McLandress, C.: Impact of the Doppler Spread Parameterization on the simulation of the middle atmosphere circulation using the MA/ECHAM4 general circulation model, J. Geophys. Res., 102, 25 751-25 762, 1997.

McFarlane, N. A.: The effect of orographically exited gravity wave drag on the general circulation of the lower stratosphere and troposphere, J. Atmos. Sci., 44, 1775-1800, 1987.

Morcrette, J. J.: Radiation and cloud radiative properties in the European Center for Medium-Range Weather Forecasts forecasting system, J.Geophys.Res., 96, 9121-9132, 1991.

Mote, P. W., Dunkerton, T. J., McIntyre, M. E., Ray, E. A., Haynes, P. H., and Russell, J. M.: Vertical velocity, vertical diffusion, and dilution by midlatitude air in the tropical lower stratosphere, J. Geophys. Res., 103, 8651-8666, 1998.

Müller, J.-F. and Brasseur, G.: IMAGES: A three-dimensional chemical trasport model of the global troposphere, J.Geophys.Res., 100, 16445-16490, 1995.

Nagashima, T., Takahashi, M., Takigawa, M., and Akiyoshi, H.: Future development of the ozone layer calculated by a general circulation model with fully interactive chemistry, Geoph. Res. Lett., 29, doi:10.1029/2001GL014026, 2002.

Ozolin, Y.: Modelling of durinal variations of gas species in the atmosphere and durinal averaging in photochemical models, Izv. Akad. Nauk, Phys. Atmos. Ocean, 28, N12, 135-143, 1992.

Pawson, S., Kodera, K., Hamilton, K., et al.: The GCM-reality intercomparison project for SPARC (GRIPS): Scientific issues and initial results, Bull. Amer. Meteor. Soc., 81,781-796, 2000.

Prather, M. J.: Numerical Advection by Conservation of SecondOrder Moments, J. Geophys. Res., 91, 6671-6681, 1986.
Roeckner, E., Arpe, K., Bengtsson, L., Christoph, M., Claussen, M., Dümenil, L., Esch, M., Giorgetta, M., Schlese, U., and Schulzweida, U.: The atmospheric general circulation model ECHAM4: Model description and simulation of the present day climate, Tech.Rep. 218, Max Planck Ins. for Meteorol., Hamburg, Germany, 1996a.

Roeckner, E., Oberuber, J. M., Bacher, A., Christoph, M., and Kirchner, I.: ENSO variability and atmospheric response in a global coupled atmosphere-ocean GCM, Clim. Dynamics., 12, 734-754, 1996b.

Roeckner, E., Brokopf, R., Esch, M., Giorgetta, M., Hagemann, S., Kornblueh, L., Manzini, E., Schlese, U., and Schulzweida, U.: The atmospheric general circulation model ECHAM5 Part II: Sensitivity of simulated climate to horizontal and vertical resolution, Tech.Rep. 354, MPI. for Meteorol., Hamburg, Germany, 2004.

Rozanov, E. V., Schlesinger, M. E., Zubov, V. A., Yang, F., and Andronova, N. G.: The UIUC three-dimensional stratospheric chemical transport model: Description and evaluation of the simulated source gases and ozone, J. Geophys. Res., 104, $11755-$ $11781,1999$.

Rozanov, E. V., Schlesinger, M. E., and Zubov, V. A.: The University of Illinois, Urbana-Champaign three-dimensional stratosphere-troposphere general circulation model with interactive ozone photochemistry: Fifteen-year control run climatology, J. Geophys. Res., 106, 27 233-27 254, 2001.

Rozanov, E. V., Schlesinger, M. E., Andronova, N. G., Yang, F., Malyshev, S. L., Zubov, V. A., Egorova, T. A., and Li, B.: Climate/chemistry effects of the Pinatubo volcanic eruption simulated by the UIUC stratosphere/troposphere GCM with interactive photochemistry, J. Geophys. Res., 107(D21), 4594, doi:10.1029/2001JD000974, 2002a.

Rozanov, E., Egorova, T., Fröhlich, C., Haberreiter, M., Peter, T., and Schmutz, W.: Estimation of the ozone and temperature sensitivity to the variation of spectral solar flux, in: From Solar Min to Max: Half a Solar Cycle with SOHO, ESA SP-508, 181-184, 2002 b.

Rozanov, E. V., Schlesinger, M. E., Egorova, T. A., et al.: Atmospheric Response to the Observed Increase of Solar UV Radiation from Solar Minimum to Solar Maximum Simulated by the UIUC Climate-Chemistry Model, J. Geophys. Res., 109, D01110, doi:10.1029/2003JD003796, 2004.

Sander, S. P., Friedl, R. R., DeMore, W. B., Golden, D. M., Hampson, R. F., Kurylo, M. J., Huie, R. E., Moortgat, G. K., Ravishankara, A. R., Kolb, C. E., and Molina, M. J.: Chemical Kinetics and Photochemical Data for Use in Stratospheric Modeling: Supplement to Evaluation 12: Update of Key Reactions, Evaluation 13, JPL Publication, 2000.

Schnadt, C., Dameris, M., Ponater, M., Hein, R., Grewe, V., and. Steil, B.: Interaction of atmospheric chemistry and climate and its impact on stratospheric ozone, Clim. Dynamics, 18, 507-517, 2002.

Sherman, A. H. and Hindmarsh, A. C.: GEARS: A package for the solution of sparse stiff, ordinary differential equations, Lawrence Livermore Lab. Rep. UCID-30114, 1980.

Shindell, D. T., Rind, D., and Lonergan, P.: Increased polar stratospheric ozone losses and delayed eventual recovery owing to increasing greenhouse-gas concentrations, Nature, 392, 589-592, 1998. 
Simmons, A., Burridge, D., Jarraud, M., Girard, C., and Wergen, W.: The ECMWF medium-range prediction models: Development of the numerical formulations and the impact of increased resolution, Meteor. Atmos. Phys., 40, 28-60, 1989.

SPARC: SPARC Intercomparison of Middle Atmosphere Climatologies, SPARC Rep. 3, 96, 2002.

Steil, B., Bruhl, C., Manzini E., et al.: A new interactive chemistryclimate model: 1. Present-day climatology and interannual variability of the middle atmosphere using the model and 9 years of HALOE/UARS data, J. Geophys. Res., 108(D9), 4290, doi:10.1029/2002JD002971, 2003.

Stott, P. and Harwood, R.: An implicit time-stepping scheme for chemical species in a global atmospheric circulation model, Ann. Geophys., 11, 377-388, 1993.

Struthers, H., Kreher, K., Austin, J., Schofield, R., Bodeker, G. E., Johnston, P. V., Shiona, H., and Thomas, A.: Past and future simulations of $\mathrm{NO}_{2}$ from a coupled chemistry climate model in comparison with observations, Atmos. Chem. Phys., 4, 22272239, 2004,

SRef-ID: 1680-7324/acp/2004-4-2227.
Swinbank, R. and Ortland, D. A.: Compilation of wind data for the UARS Reference Atmosphere Project, J. Geophys. Res., 108(D19), 4615, doi:10.1029/2002JD003135, 2003.

Takigawa, M., Takahashi, M., and Akiyoshi, H.: Simulation of ozone and other chemical species using a Center for Climate System Research/National Institute for Environmental Studies atmospheric GCM with coupled stratospheric chemistry, J. Geophys. Res., 104, 14 003-14 018, 1999.

Thompson, D. and Wallace, J.: The arctic oscillation signature in the wintertime geopotential height and temperature fields, Geophys. Res. Lett., 25, 1297-1300, 1998.

Zubov, V., Rozanov, E., and Schlesinger, M.: Hybrid scheme for tree-dimensional advective transport, Mon. Wea. Rev., 127, 1335-1346, 1999.

Williamson, D. L. and Rasch, P. J.: Two-dimensional semilagrangian transport with shape-preserving interpolation, Mon. Weather Rev., vol. 117, no. 1, 102-129, 1989. 